\title{
Adaptive Consensus of Distributed Varying Scale Wireless Sensor Networks under Tolerable Jamming Attacks
}

\author{
Jinping Mou \\ School of Mathematics and Information Engineering, Taizhou University, Linhai 317000, China \\ Correspondence should be addressed to Jinping Mou; mjptougaozhuanyong@163.com
}

Received 16 August 2013; Accepted 16 December 2013

Academic Editor: Kwok-Wo Wong

Copyright (C) 2013 Jinping Mou. This is an open access article distributed under the Creative Commons Attribution License, which permits unrestricted use, distribution, and reproduction in any medium, provided the original work is properly cited.

\begin{abstract}
Consensus problem is investigated for a varying scale wireless sensor network (VSWSN) under tolerable jamming attacks, where the scale of the network is increasing or decreasing due to the newly joined nodes or the removed nodes, respectively; the tolerable jamming attack means that the attack strength is limited. It supposes that during the communications, all nodes may encounter with the tolerable jamming attacks; when the attack power is larger than the given value, the attacked nodes fall asleep, or otherwise the nodes are awakened. Under the sleep method, based on the Lyapunov method, it shows that if the communicating graph is the global limited intersectional connection (GLI connection) and the system has the enough dwell time in the intersectional topology, then under the designed consensus protocol, all nodes achieve the global average consensus.
\end{abstract}

\section{Introduction}

In the past decades, distributed coordination of wireless sensor network (WSN) has been widely investigated, such as formation control, target-tracking, and environmental monitoring $[1,2]$. For the distributed coordination, consensus is the fundamental requirement in which all states of sensors achieve a common value, such as the average consensus [3], and sample data-based consensus $[4,5]$. The characteristics of WSN including the unreliable links and the limited energy supply render the challenges of developing algorithms and optimizing topology to achieve the consensus control; therefore, many topology optimization and algorithm development problems have been studied.

The early consensus work can be found in [6], where the general methods of consensus control are proposed. In recent years, consensus problems coupling with optimizing topology have been investigated. For instance, under a leaderfollowing framework, the consensus problems were studied $[7,8]$. More details can be found in [9-12]. Based on the sleeping-awaking method, consensus problem of the Markovian switching WSN with multiple time delays was studied [13]. Based on the stochastic matrices, the consensus algorithm was proposed in [14]; more results are proposed in $[15,16]$.

Recently, adaptive consensus problem has attracted much attention. For instance, a distributed consensus protocol with an adaptive law was proposed by adjusting the coupling weights [17]. According to iterative learning method, an adaptive consensus protocol was designed for all follower agents to track a leader [11]. More results are shown in $[18,19]$.

Notice that most of the above results on the consensus are associated with the fixed node set. However, in the real applications, the scale of WSN often is varying due to the node removal or the new nodes joining the network, where the node removal means that some nodes quit from the network because the energy is exhausted.

In recent years, the related consensus problems of the varying scale networks (VSNs) have risen researchers attentions, such as consensus of the scale-free network (SFN), where degree distribution follows a power law, at least asymptotically [20,21]. In the literature [22], consensus problem of varying scale wireless sensor network (VSWSN) was investigated, where the varying topology of VSWSN is expressed 
by the node attached component sequences. As a result, the global limited intersectional connection (GLI connection) is the necessary condition of system achieving the global consensus.

\section{Related Work}

In fact, networks often encounter with some attacks, such as jamming attacks, tampering attacks, and exhaustion attacks. Under some attacks, the networks may be broken down, the coordinative behavior cannot be kept, and all nodes cannot achieve the consensus. In the literature [23], the synchronization against the removed nodes of complex dynamical networks was studied, where the communications are based on the switching topology. In recent years, consensus problem with the attacks has attracted some researchers attention. In [24], Wang et al. studied consensus problem of networks under the recoverable attacks, where after being attacked, the system becomes paralyzed; in the next period, the system recovers and achieves the consensus, and the relation between the state of system and the attack signal is not considered.

However, in real applications, the system often is influenced by the attack signals whatever the topology is optimized. Namely, the dynamic state of the system is impacted by the attack signals, and up to now, consensus problem of VSWSNs with the tolerable jamming attack has not attracted much attention.

The main contribution of this paper is to investigate the consensus problem of VSWSN under the tolerable jamming attacks. It begins with the introduction on communicating graph. Namely, all nodes communicate information among the components; the communications among all different node attached components are based on the intersectional topologies. Then the states of all components can be described by the different stochastic equations (DSEs); the consensus can be regarded that all trivial solutions of DSEs converge to the same value. The aim of this paper is to establish some criteria of VSWSN under the tolerable jamming attacks.

It should point out that the introduced topology in this paper is different from the previous results. In most literatures, such as the node set of network is fixed, system switches among the different spinning trees, or system communicates information in the union connected topology, and the related results cannot be applied for VSWSN because of the fixed scale. In fact, whatever VSWSN runs sleeping algorithm in any surroundings, for example, system encounters with attacks, the topology can be expressed by the node attached sequences, the connectivity of the network can be shown by local limited intersectional connection (LLI connection) or GLI connection, and the general connection is the special case of GLI connected.

The outline of this paper is listed as follows. In Section 3, some basic concepts, notations, and problem formulation are introduced. In Section 4, the main results are proposed. In Section 5, a numeral example shows the reliability of the proposed results. In Section 6, several conclusions are obtained.

\section{Preliminaries}

\subsection{Notations and Some Conceptions}

Notations. Throughout this paper, $\aleph=\{\overline{0}, \overline{1}, \overline{2}, \ldots, \bar{\kappa}, \ldots\}$ denotes the topology set of the varying scale wireless sensor network (VSWSN); the elements of the set satisfy the following partial sequence:

$$
\overline{0} \preceq \overline{1} \preceq \overline{2} \preceq \cdots \preceq \bar{\kappa}_{1} \preceq \bar{\kappa}_{2} \preceq \cdots,
$$

where the listed topologies will appear in succession, $\overline{0}$ is the initial topology, and $\bar{\kappa}_{1}, \bar{\kappa}_{2}$ are called the adjacent topologies. Accordingly, $\left[t_{\bar{\kappa}_{1}}, t_{\bar{\kappa}_{2}}\right)$ denotes the dwell time interval of topology $\bar{\kappa}_{1}$; if $\bar{\kappa}_{1} \neq \bar{\kappa}_{2}$, then $\bar{\kappa}_{1} \prec \bar{\kappa}_{2}$ denotes the relation between $\bar{\kappa}_{1}$ and $\bar{\kappa}_{2}$.

In order to express the varying topology, a discernible function $\theta: T \rightarrow \aleph$ is introduced; $\theta(t)=\bar{\kappa}_{1} \in \aleph$, where $t \in\left[t_{\bar{\kappa}_{1}}, t_{\bar{\kappa}_{2}}\right)$. According to $\theta(t)$, the varying topology of VSWSN can be denoted by a varying graph $G(\theta(t))=G\left(\bar{\kappa}_{1}\right)=$ $\left(V\left(\bar{\kappa}_{1}\right), E\left(\bar{\kappa}_{1}\right), A\left(\bar{\kappa}_{1}\right)\right)$, where $V\left(\bar{\kappa}_{1}\right)=V_{1}\left(\bar{\kappa}_{1}\right) \cup V_{2}\left(\bar{\kappa}_{1}\right)$ denotes the varying node set, $V_{1}\left(\bar{\kappa}_{1}\right)=\left\{1_{\bar{\kappa}_{1}}, 2_{\bar{\kappa}_{1}}, \ldots, i_{\bar{\kappa}_{1}}, \ldots, \alpha_{\bar{\kappa}_{1}}\right\}$ refers to the valid node set of which all elements inherit from the former topology, $V_{2}\left(\bar{\kappa}_{1}\right)=\left\{1_{\bar{\kappa}_{1}}^{\prime}, 2_{\bar{\kappa}_{1}}^{\prime}, \ldots, \beta_{\bar{\kappa}_{1}}^{\prime}\right\}$ is the newly joined node set, and $E\left(\bar{\kappa}_{1}\right)=\left\{\left(i_{\bar{\kappa}_{1}}, j_{\bar{\kappa}_{1}}\right) \mid i_{\bar{\kappa}_{1}} \neq j_{\bar{\kappa}_{1}}, i_{\bar{\kappa}_{1}}, j_{\bar{\kappa}_{1}} \in\right.$ $\left.V\left(\bar{\kappa}_{1}\right)\right\}$ stands for the edge set.

$N_{i}(\bar{\kappa})=\left\{j_{\bar{\kappa}} \mid i_{\bar{\kappa}} \neq j_{\bar{\kappa}}, i_{\bar{\kappa}}, j_{\bar{\kappa}} \in V(\bar{\kappa})\right\}$ refers to the neighbor set of node $i_{\bar{\kappa}}$ in the topology $\bar{\kappa} . A(\bar{\kappa})=\left(a_{i j}(\bar{\kappa})\right)_{w_{\bar{\kappa}}} \in R^{w_{\bar{\kappa}} \times w_{\bar{\kappa}}}$ stands for the weighted symmetric matrix, where $w_{\bar{\kappa}}=\alpha_{\bar{\kappa}}+$ $\beta_{\bar{\kappa}}, a_{i j}(\bar{\kappa})$ takes value in 0 or $1 . \forall j_{\bar{\kappa}} \in N_{i}(\bar{\kappa}), a_{i j}(\bar{\kappa})=1$ means that there exists information flow between the awaking nodes $j_{\bar{\kappa}}$ and $i_{\bar{\kappa}}$; if one of $j_{\bar{\kappa}}$ or $i_{\bar{\kappa}}$ is asleep, then $a_{i j}(\bar{\kappa})=0$; if $j_{\bar{\kappa}} \notin$ $N_{i}(\bar{\kappa})$, then $a_{i j}(\bar{\kappa}) \equiv 0$.

$L\left(w_{\bar{\kappa}}\right)=\left(l_{i j}(\bar{\kappa})\right)_{w_{\bar{\kappa}} \times w_{\bar{\kappa}}}$ is the Laplacian matrix, and $l_{i_{\bar{\kappa}} j_{\bar{\kappa}}}$ is defined by

$$
l_{i j}(\overline{\mathcal{K}})= \begin{cases}\sum_{j_{\bar{\kappa}}} a_{i j}(\overline{\mathcal{K}}), & i_{\overline{\mathcal{\kappa}}}=j_{\overline{\mathcal{\kappa}}}, j_{\overline{\mathcal{\kappa}}} \in N_{i}(\overline{\mathcal{K}}), \\ -a_{i j}(\overline{\mathcal{K}}), & i_{\overline{\mathcal{K}}} \neq j_{\overline{\mathcal{K}}}, j_{\overline{\mathcal{K}}} \in N_{i}(\overline{\mathcal{\kappa}}) .\end{cases}
$$

The following conceptions are used frequently [22].

Definition 1. $\forall i_{\bar{\kappa}} \in V(\bar{\kappa})$; if there exists a component $C_{i}^{\prime}(\bar{\kappa})$ of $G(\bar{\kappa})$ such that $i_{\bar{\kappa}} \in V_{i}^{\prime}(\bar{\kappa})$, then $C_{i}^{\prime}(\bar{\kappa})$ is said to be the node attached component of $i_{\bar{\kappa}}$; if there exists the sequence $C_{i}^{\prime}\left(\kappa_{1}\right), C_{i}^{\prime}\left(\kappa_{2}\right), \ldots$ such that $i_{\bar{\kappa}} \in V_{i}^{\prime}\left(\kappa_{1}\right), i_{\bar{\kappa}} \in V_{i}^{\prime}\left(\kappa_{2}\right) \ldots$, then sequence $C_{i}^{\prime}\left(\kappa_{1}\right), C_{i}^{\prime}\left(\kappa_{2}\right), \ldots$ is said to be the node attached component sequence of node $i_{\bar{\kappa}}$.

$C_{i}^{\prime}(\bar{\kappa})$ denotes a component of $G(\bar{\kappa})$, where $C_{i}^{\prime}(\bar{\kappa})=$ $\left\{V_{i}^{\prime}(\bar{\kappa}), A_{i}^{\prime}(\bar{\kappa}), E_{i}^{\prime}(\bar{\kappa})\right\}, i_{\bar{\kappa}} \in V_{i}^{\prime}(\bar{\kappa}) \subset V(\bar{\kappa}), E_{i}^{\prime}(\bar{\kappa}) \subset E(\bar{\kappa})$. $V_{i}^{\prime}(\overline{\mathcal{\kappa}})=V_{i}^{\prime 1}(\overline{\mathcal{\kappa}}) \cup V_{i}^{\prime 2}(\overline{\mathcal{\kappa}})$, where $V_{i}^{\prime 1}(\overline{\mathcal{\kappa}})$ refers to the valid node set of which all elements inherit from the former attached component of $i_{\bar{\kappa}}, V_{i}^{\prime 2}(\bar{\kappa})$ is the newly joined node set.

Definition 2. $\forall i_{\bar{\kappa}}, j_{\bar{\kappa}} \in V(\bar{\kappa})$; if there exists two related attached component sequences $C_{i}^{\prime}\left(\bar{\kappa}_{1}\right), C_{i}^{\prime}\left(\bar{\kappa}_{2}\right), \ldots, C_{i}^{\prime}\left(\bar{\kappa}_{r}\right), \ldots$ and $C_{j}^{\prime \prime}\left(\bar{\kappa}_{1}\right), C_{j}^{\prime \prime}\left(\bar{\kappa}_{2}\right), \ldots, C_{j}^{\prime \prime}\left(\bar{\kappa}_{r}\right), \ldots$, respectively, and if there exists $\bar{\kappa}_{0} \in \aleph$ and $\aleph^{\prime}=\left\{\bar{\kappa}_{r_{1}}, \bar{\kappa}_{r_{2}}, \ldots\right\} \subset \aleph$, such that 
$C_{i}^{\prime}\left(\bar{\kappa}_{r}\right)=C_{j}^{\prime \prime}\left(\bar{\kappa}_{r}\right)$, where $\bar{\kappa}_{0} \preceq \cdots \preceq \bar{\kappa}_{r} \preceq \cdots$, then the communicating graph is said to be the global limited intersectional connection (GLI connection), and $\bar{\kappa}_{r}$ is called the intersectional topology.

Assumption 3. After the intersectional topology, the node set may be varied. For example, let $\bar{\kappa}_{1}$ be the intersectional topology, and $\bar{\kappa}_{2}$ is the next topology of it; in $\left[t_{\bar{\kappa}_{1}}, t_{\overline{\mathcal{K}}_{2}}\right)$, all nodes will not be removed, but at time $t_{\bar{\kappa}_{2}}$, some new nodes will be removed and some nodes will be added.

Remark 4. Under Assumption 3, it follows that between every two adjacent intersectional topologies $\bar{\kappa}_{r}$ and $\bar{\kappa}_{r}^{\prime}$, each node $i_{\bar{\kappa}}$ will appear in $\bar{\kappa}_{r}^{\prime}$, where $\bar{\kappa}_{r}<\bar{\kappa} \preceq \bar{\kappa}_{r}^{\prime}$. $\bar{\kappa}_{1} \neq \bar{\kappa}_{2}$.

$i_{\bar{\kappa}_{1}}$ and $i_{\bar{\kappa}_{2}}$ refer to a node in the different topology, where

3.2. Problem Statement. In many applications, the communication topology of VSWSN is based on the multiple components. In this paper, the communication is the componentbased.

For $i_{\bar{\kappa}} \in V(\bar{\kappa})$, let $x_{i}(t, \bar{\kappa})$ be the state of sensor $i_{\bar{\kappa}}$, where $x_{i}(t, \bar{\kappa}) \in R$. Suppose the state of $i_{\bar{\kappa}}$ is given by

$$
\dot{x}_{i}(t, \bar{\kappa})=u_{i_{\bar{\kappa}}}(\mathrm{t}),
$$

where $u_{i_{\bar{\kappa}}}(t)$ is the consensus protocol, and it is given by

$$
u_{i_{\bar{\kappa}}}(t)=\varepsilon_{\bar{n}_{\bar{\kappa}}} \sum_{j_{\bar{\kappa}} \in N_{i}(\bar{\kappa})} a_{i j}(\bar{\kappa})\left[y_{j}(t, \bar{\kappa})-x_{i}(t, \bar{\kappa})\right], \quad t \in\left[t_{\bar{\kappa}}, t_{\bar{\kappa}^{\prime}}\right),
$$

$\bar{\kappa}^{\prime}$ is the next topology of $\bar{\kappa}, y_{j}(t, \bar{\kappa})$ is the state of $j_{\bar{\kappa}}$ that is measured by $i_{\bar{\kappa}}, y_{j}(t, \bar{\kappa})=x_{j}(t, \bar{\kappa})+f_{i j}(t)+w_{i j}(t), f_{i j}(t)$ is the measured attack signal, $f_{i j}(t)=f_{j i}(t)$, and $w_{i j}(t)$ is the white noise.

Consider $\forall C_{i}^{\prime}(\bar{\kappa}) \subset G(\bar{\kappa})$; based on the dynamic (3) and protocol (4), the dynamic state of component $C_{i}^{\prime}(\bar{\kappa})$ which attaches on node $i_{\bar{\kappa}}$ is described by

$$
\begin{array}{r}
\dot{X}_{\widehat{n}_{\bar{\kappa}}}^{i}(t)=-\varepsilon_{\widehat{n}_{\bar{\kappa}}} L_{\widehat{n}_{\bar{\kappa}}}^{i} X_{\bar{n}_{\bar{\kappa}}}^{i}(t)+\varepsilon_{\widehat{n}_{\bar{\kappa}}} \Gamma^{i}\left[F_{\widehat{n}_{\bar{\kappa}}}^{i}(t)+W_{\widehat{n}_{\bar{\kappa}}}^{i}(t)\right], \\
i_{\overline{\mathcal{K}}} \in V_{1}^{i}(\overline{\mathcal{K}}), t \in\left[t_{\overline{\mathcal{K}}}, t_{\overline{\bar{\kappa}}^{\prime}}\right),
\end{array}
$$

where $L_{\hat{n}_{\bar{\kappa}}}^{i}=\left(l_{i_{\bar{\kappa}} j_{\bar{\kappa}}}\right)_{\hat{n}_{\bar{\kappa}} \times \widehat{n}_{\bar{\kappa}}}, X_{\hat{n}_{\bar{\kappa}}}^{i}(k)=\left[X_{p_{\bar{\kappa}}}^{i}(k)^{T}, X_{q_{\bar{\kappa}}^{\prime}}^{i}(k)^{T}\right]^{T}$, $\widehat{n}_{\bar{\kappa}}=p_{\bar{\kappa}}+q_{\bar{\kappa}}, X_{p_{\bar{\kappa}}}(t)$ refers to the state vector of node set $V_{1}^{i}(\bar{\kappa})=\left\{1_{\bar{\kappa}}, 2_{\bar{\kappa}}, \ldots, i_{\bar{\kappa}}, \ldots, p_{\bar{\kappa}}\right\} \subseteq V_{1}(\bar{\kappa}), p_{\bar{\kappa}} \leq \alpha_{\bar{\kappa}}, X_{q_{\bar{\kappa}}^{\prime}}^{i}(k)$ is the state vector of the newly joined node set $V_{2}^{i}(\bar{\kappa})=$ $\left\{1_{\overline{\mathcal{K}}}^{\prime}, 2_{\overline{\mathcal{K}}}^{\prime}, \ldots, i_{\overline{\mathcal{K}}}^{\prime}, \ldots, q_{\overline{\mathcal{K}}}^{\prime}\right\}, q_{\bar{\kappa}} \leq \beta_{\bar{\kappa}}$, and

$$
\begin{array}{r}
X_{p_{\bar{\kappa}}}^{i}(t)=\left[x_{1_{\bar{\kappa}}}(t, \bar{\kappa}), \ldots, x_{i}(t, \bar{\kappa}), \ldots, x_{p}(t, \bar{\kappa})\right]^{T}, \\
i_{\bar{\kappa}} \in V_{1}^{i}(\bar{\kappa}),
\end{array}
$$

$$
\begin{gathered}
X_{q_{\bar{\kappa}}^{\prime}}^{i}(t)=\left[x_{1^{\prime}}(t, \bar{\kappa}), \ldots, x_{i^{\prime}}(t, \bar{\kappa}), \ldots, x_{q^{\prime}}(t, \bar{\kappa})\right]^{T}, \\
i_{\bar{\kappa}}^{\prime} \in V_{2}^{i}(\bar{\kappa}), \\
\Gamma^{i}=\operatorname{diag}\left\{\beta_{1}^{T}, \beta_{2}^{T}, \ldots, \beta_{i}^{T}, \ldots, \beta_{\bar{n}_{\bar{\kappa}}}^{T}\right\}, \\
\beta_{i}^{T}=\left(a_{i 1}(\bar{\kappa}), a_{i 2}(\bar{\kappa}), \ldots, a_{i \hat{n}_{\bar{\kappa}}}(\bar{\kappa})\right), \\
W^{i}(t)=\operatorname{diag}\left\{W_{t}^{1}, W_{t}^{2}, \ldots, W_{t}^{i}, \ldots, W_{t}^{\widehat{n}_{\bar{\kappa}}}\right\}, \\
W_{t}^{i}=\left(w_{i 1}(t), w_{i 2}(t), \ldots, w_{i j}(t), w_{i \bar{n}_{\bar{\kappa}}}(t)\right)^{T}, \\
E\left[w_{i j}(t)\right]=0, \quad E\left[w_{i j}(t) w_{i j}(t)^{T}\right]=1, \\
F_{\hat{n}_{\bar{\kappa}}}^{i}(t)=\operatorname{diag}\left\{F^{1}(t), F^{2}(t), \ldots, F^{i}(t), \ldots, F^{\hat{n}_{\bar{\kappa}}}(t)\right\}, \\
F^{i}(t)=\left(f_{i 1}(t), f_{i 2}(t), \ldots, f_{i j}(t), f_{i \widehat{n}_{\bar{\kappa}}}(t)\right)^{T} .
\end{gathered}
$$

Remark 5. Analogously, if $\widehat{n}_{\bar{\kappa}}$ is substituted by $w_{\bar{\kappa}}$, then system (5) refers to the whole system. Namely,

$$
\begin{array}{r}
\dot{X}_{w_{\bar{\kappa}}}(t)=-\varepsilon_{w_{\bar{\kappa}}} L_{w_{\bar{\kappa}}} X_{w_{\bar{\kappa}}}(t)+\varepsilon_{w_{\bar{\kappa}}} \Gamma\left[F_{w_{\bar{\kappa}}}(t)+W_{w_{\bar{\kappa}}}(t)\right], \\
i_{\bar{\kappa}} \in V_{1}(\bar{\kappa}), t \in\left[t_{\bar{\kappa}^{\prime}}, t_{\bar{\kappa}^{\prime}}\right) .
\end{array}
$$

Consider $\forall i_{\bar{\kappa}} \in V_{1}^{i}(\bar{\kappa})$; let $e_{j}(t, \bar{\kappa})=x_{j}(t, \bar{\kappa})-x_{i}^{0}(t, \bar{\kappa})$, $e(t, \bar{\kappa})=X(t, \bar{\kappa})-x_{i}^{0}(t, \bar{\kappa}) \otimes 1_{\bar{n}_{\bar{\kappa}}}$, it obtains the systematic error of (5) as follows:

$$
e^{i}(t, \bar{\kappa})=X_{\widehat{n}_{\bar{\kappa}}}^{i}(t)-\mathbf{1}_{\widehat{n}_{\bar{\kappa}}} \otimes x_{i}^{0}(t, \bar{\kappa})=\Phi_{\bar{\kappa}} X_{\widehat{n}_{\bar{\kappa}}}^{i}(t),
$$

where

$$
\begin{gathered}
x_{i}^{0}(t, \bar{\kappa})=\frac{1}{\widehat{n}_{\bar{\kappa}}} \sum_{i_{\bar{\kappa}} \in V_{i}(\bar{\kappa})} x_{i}(t, \bar{\kappa}), \\
e^{i}(t, \bar{\kappa})=\left(e_{1}(t, \bar{\kappa}), \ldots, e_{i}(t, \bar{\kappa}), \ldots, e_{\widehat{n}}(t, \bar{\kappa})\right)^{T}, \\
\Phi=I_{\bar{n}_{\bar{\kappa}}}-\frac{1}{\widehat{n}_{\overline{\mathcal{\kappa}}}} \times \mathbf{1}_{\widehat{n}_{\bar{\kappa}} \times \widehat{n}_{\bar{\kappa}}},
\end{gathered}
$$

where $\mathbf{1}_{\widehat{n}_{\bar{\kappa}} \times \widehat{n}_{\bar{\kappa}}}$ is the $\widehat{n}_{\bar{\kappa}} \times \widehat{n}_{\bar{\kappa}}$ matrix in which each entry is 1 .

From (8), one gets

$$
\begin{aligned}
\dot{e}^{i}(t, \bar{\kappa})= & \Phi_{\bar{\kappa}}^{i} \dot{X}_{\widehat{n}_{\bar{\kappa}}}^{i}(t)=\Phi_{\bar{\kappa}}^{i}\left(-\varepsilon_{\bar{\kappa}}^{i} L_{\widehat{n}_{\bar{\kappa}}}\right) X_{\widehat{n}_{\bar{\kappa}}}^{i}(t) \\
& +\Phi_{\bar{\kappa}_{\mathcal{n}_{\bar{\kappa}}}}^{i} \Gamma^{i}\left[F_{\widehat{n}_{\bar{\kappa}}}^{i}(t)+W_{\widehat{n}_{\bar{\kappa}}}^{i}(t)\right] .
\end{aligned}
$$

Assumption 6. Suppose that each node can sense the strength of the attack signal in its perceivable areas; in terms of carrier sense of ASCENT algorithm [25], every sensor is awake or asleep according to the attacks, namely,

$$
a_{i j}(\bar{\kappa})= \begin{cases}1, & \left\|f_{i j}(t)\right\| \leq \frac{\alpha(\bar{\kappa})\left\|x^{i}(t, \bar{\kappa})\right\|}{d_{\bar{\kappa}}^{i}}, \\ 0, & \text { otherwise }\end{cases}
$$

where $\alpha(\bar{\kappa})$ is the constant and $d_{\bar{\kappa}}^{i}$ is the maximal degree of component which is attached by node $i_{\bar{\kappa}}$. 
Assumption 7. Under criterion (11), the topology of VSWSN is GLI connection.

Definition 8. For VSWSN (7), the jamming attacks are said to be the tolerable if VSWSN (7) satisfies Assumption 7.

Definition 9. Consider $\forall C_{i}^{\prime}(\bar{\kappa}) \subset G(\bar{\kappa})$ and $\forall i_{\bar{\kappa}}, q_{\bar{\kappa}} \in V_{i}(\bar{\kappa})$; if

$$
\lim _{t \rightarrow \infty} E\left(\left\|x_{i}(t, \bar{\kappa})-x_{q}(t, \bar{\kappa})\right\|^{2}\right)=0
$$

then VSWSN (5) is said to achieve the component consensus. In addition, if $C_{i}^{\prime}(\bar{\kappa})=G(\bar{\kappa})$, and (12) holds, then VSWSN (5) is said to achieve the global consensus.

If $\lim _{t \rightarrow \infty} E\left(\left\|x_{i}(t, \bar{\kappa})-x_{i}^{0}(t, \bar{\kappa})\right\|^{2}\right)=0$, then VSWSN (5) is said to achieve the component average consensus. In addition, if $C_{i}^{\prime}(\overline{\mathcal{\kappa}})=G(\overline{\mathcal{\kappa}})$ and (12) holds, then VSWSN (5) is said to achieve the global average consensus.

In the following section, the consensus problem under the tolerable attacks is investigated via the error system (10).

Remark 10. According to Assumption 3, if VSWSN is GLIconnected, then each intersectional topology is connected, and it holds that $\sum_{j_{\bar{\kappa}} \in N_{i_{\bar{\kappa}}}} a_{i j}(\bar{\kappa})>0$. According to the literature [26], all eigenvalues of $-L_{\widehat{n}_{\bar{\kappa}}}^{i}$ satisfy

$$
0=\lambda^{1}(\bar{\kappa})>\lambda^{2}(\bar{\kappa}) \geq \cdots \geq \lambda^{\hat{n}_{\bar{\kappa}}}(\bar{\kappa}) \geq-2 \Delta(\overline{\mathcal{\kappa}}),
$$

where

$$
\Delta(\bar{\kappa})=\max \left\{\sum_{j_{\bar{\kappa}} \in N_{i_{\bar{\kappa}}}} a_{i j}(\bar{\kappa}) \mid i_{\bar{\kappa}} \in V(\bar{\kappa})\right\} .
$$

For convenience, $\lambda^{i j}(\bar{\kappa})$ denotes the second largest eigenvalue of $-L\left(w_{\bar{\kappa}}\right), i_{\bar{\kappa}}, j_{\bar{\kappa}} \in V(\bar{\kappa}), \lambda^{i}(\bar{\kappa})$ refers to the second largest eigenvalue of $-L\left(\widehat{n}_{\bar{\kappa}}\right), i_{\bar{\kappa}} \in V(\bar{\kappa})$, and $j_{\bar{\kappa}} \notin V(\bar{\kappa})$.

Remark 11. If a sensor leaves from its neighbors and becomes an isolated node, its state may not keep in coordination with other nodes temporarily. Note that if the communicating graph is GLI-connected, the node has a chance to communicate with other nodes and keep coordination with other nodes.

Remark 12. In model (5), the state of each node may be influenced by the attack signal function $F^{i}(t)$.

Remark 13. System (5) achieves the component average consensus refers that to the fact that the norm of $e^{i}(t, \bar{\kappa})$ converges to zero. Similarly, if $C_{i}^{\prime}(\bar{\kappa})=G(\bar{\kappa})$, then the global average consensus means that the norm of $e(t, \bar{\kappa})$ converges to zero.

\section{Main Results}

This section will investigate the consensus problem while the system encounters with the jamming attacks. The aim of this section is to establish some consensus criteria of VSWSN.
Let

$$
\begin{aligned}
\delta^{i j}(t, \bar{\kappa}) & =\frac{1}{2}\left[e^{i j}(t, \bar{\kappa})^{T} e^{i j}(t, \bar{\kappa})\right], \\
\delta^{i}(t, \bar{\kappa}) & =\frac{1}{2} e^{i}(t, \bar{\kappa})^{T} e^{i}(t, \bar{\kappa}), \\
\delta^{j}(t, \bar{\kappa}) & =\frac{1}{2} e^{j}(t, \bar{\kappa})^{T} e^{j}(t, \bar{\kappa}),
\end{aligned}
$$

where $e^{i j}(t, \bar{\kappa})=\left(e_{1}(t, \bar{\kappa})^{T}, \ldots, e_{i}(t, \bar{\kappa})^{T}, \ldots, e_{j}(t, \bar{\kappa})^{T}, \ldots\right.$, $\left.e_{\widehat{n}}(t, \bar{\kappa})^{T}\right)$, then the update laws of $\varepsilon_{\widehat{n}_{\bar{\kappa}}}(n=0,1,2, \ldots)$ are provided by

$$
\varepsilon_{\bar{n}_{\bar{\kappa}}}=c\left\{E\left[\delta^{j}\left(t_{\bar{\kappa}_{m-1}}, \bar{\kappa}_{m-1}\right)\right]+E\left[\delta^{j}\left(t_{\bar{\kappa}_{m}}, \bar{\kappa}_{m}\right)\right]\right\}
$$

where $m \geq 1$ and $c$ is a positive constant, and one proposition is obtained as follows.

Proposition 14. $\delta^{i j}(t, \bar{\kappa})$ satisfies

$$
E\left[\dot{\delta}^{i j}(t, \bar{\kappa})\right] \leq \varepsilon_{\widehat{n}_{\bar{\kappa}}}\left(\lambda_{\bar{\kappa}}^{i j} E\left[\delta^{i j}(t, \bar{\kappa})\right]+\Delta \delta^{i}\right)
$$

where $\Delta \delta^{i}=(1 / 2) E\left[F^{i}(t)^{T} \Gamma^{T} \Phi_{\bar{\kappa}}^{T} \Phi_{\bar{\kappa}} X_{\widehat{n}_{\bar{\kappa}}}(t)+X_{\widehat{n}_{\bar{\kappa}}}(t)^{T} \Phi_{\bar{\kappa}}^{T}\right.$ $\left.\Phi_{\bar{\kappa}} \Gamma F^{i}(t)\right]$.

Proof. Note that

$$
\begin{aligned}
& \dot{\delta}^{i j}(t, \bar{\kappa}) \\
& =\frac{1}{2}\left[e^{i j}(t, \bar{\kappa})^{T} e^{i j}(t, \bar{\kappa})+e^{i j}(t, \bar{\kappa})^{T} \dot{e}^{i j}(t, \bar{\kappa})\right] \\
& \leq \frac{1}{2}\left[X_{\widehat{n}_{\bar{\kappa}}}(t)^{T}\left(-\varepsilon_{\widehat{n}_{\bar{\kappa}}} L_{\widehat{n}_{\bar{\kappa}}}\right)^{T} \Phi_{\overline{\mathcal{\kappa}}}^{T}+\left(F^{i}(t)+W(t)\right)^{T} \Gamma^{T} \varepsilon_{\widehat{n}_{\bar{\kappa}}}^{T} \Phi_{\bar{\kappa}}^{T}\right] \\
& \times \Phi_{\bar{\kappa}} X_{\widehat{n}_{\bar{\kappa}}}(t)+\frac{1}{2} X_{\widehat{n}_{\bar{\kappa}}}(t)^{T} \Phi_{\bar{\kappa}}^{T}
\end{aligned}
$$

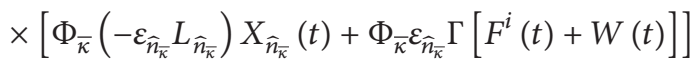

$$
\begin{aligned}
& =\frac{1}{2}\left[X_{\widehat{n}_{\bar{\kappa}}}(t)^{T}\left(-\varepsilon_{\widehat{n}_{\bar{\kappa}}} L_{\widehat{n}_{\bar{\kappa}}}\right)^{T} \Phi_{\bar{\kappa}}^{T} \Phi_{\bar{\kappa}} X_{\widehat{n}_{\bar{\kappa}}}(t)+\left(F^{i}(t)+W(t)\right)^{T}\right. \\
& \times \Gamma^{T} \varepsilon_{\bar{n}_{\bar{\kappa}}}^{T} \Phi_{\bar{\kappa}}^{T} \Phi_{\bar{\kappa}} X_{\widehat{n}_{\bar{\kappa}}}(t) \\
& +X_{\widehat{n}_{\bar{\kappa}}}(t)^{T} \Phi_{\bar{\kappa}}^{T} \Phi_{\bar{\kappa}}\left(-\varepsilon_{\widehat{n}_{\bar{\kappa}}} L_{\widehat{n}_{\bar{\kappa}}}\right) X_{\widehat{n}_{\bar{\kappa}}}(t) \\
& \left.+X_{\widehat{n}_{\bar{\kappa}}}(t)^{T} \Phi_{\bar{\kappa}}^{T} \Phi_{\bar{\kappa}} \varepsilon_{\widehat{n}_{\bar{\kappa}}} \Gamma\left(F^{i}(t)+W(t)\right)\right]
\end{aligned}
$$


then it holds that

$$
\begin{gathered}
E\left[\dot{\delta}^{i j}(t, \bar{\kappa})\right] \leq E\left[\frac{1}{2} X_{\widehat{n}_{\bar{\kappa}}}(t)^{T}\left(-\varepsilon_{\widehat{n}_{\bar{\kappa}}} L_{\widehat{n}_{\bar{\kappa}}}\right)^{T} \Phi_{\bar{\kappa}}^{T} \Phi_{\bar{\kappa}} X_{\widehat{n}_{\bar{\kappa}}}(t)\right] \\
+\frac{1}{2} E\left\{\left[X_{\widehat{n}_{\bar{\kappa}}}(t)^{T} \Phi_{\bar{\kappa}}^{T} \Phi_{\bar{\kappa}}\left(-\varepsilon_{\widehat{n}_{\bar{\kappa}}} L_{\widehat{n}_{\bar{\kappa}}}\right) X_{\widehat{n}_{\bar{\kappa}}}(t)\right]\right. \\
+F^{i}(t)^{T} \Gamma^{T} \varepsilon_{\hat{n}_{\bar{\kappa}}}^{T} \Phi_{\bar{\kappa}}^{T} \Phi_{\bar{\kappa}} X_{\hat{n}_{\bar{\kappa}}}(t) \\
\left.+X_{\widehat{n}_{\bar{\kappa}}}(t)^{T} \Phi_{\bar{\kappa}}^{T} \Phi_{\bar{\kappa}} \varepsilon_{\widehat{n}_{\bar{\kappa}}} \Gamma F^{i}(t)\right\} \\
\leq \varepsilon_{\widehat{n}_{\bar{\kappa}}}\left(\lambda_{\bar{\kappa}}^{i j} E\left[e^{i j}(t, \bar{\kappa})^{T} e^{i j}(t, \bar{\kappa})\right]+\Delta \delta^{i}\right) \\
=\varepsilon_{\widehat{n}_{\bar{\kappa}}}\left(\lambda_{\bar{\kappa}}^{i j} E\left[\delta^{i j}(t, \bar{\kappa})\right]+\Delta \delta^{i}\right) .
\end{gathered}
$$

This completes the proof.

Let

$$
\delta^{i}(t, \bar{\kappa})=\delta_{1}^{i}(t, \bar{\kappa})+\delta_{2}^{i}(t, \bar{\kappa})
$$

where

$$
\begin{gathered}
\delta_{1}^{i}(t, \bar{\kappa})=\frac{1}{2} e_{1}^{i}(t, \bar{\kappa})^{T} e_{1}^{i}(t, \bar{\kappa}), \\
\delta_{2}^{i}(t, \bar{\kappa})=\frac{1}{2} e_{2}^{i}(t, \bar{\kappa})^{T} e_{2}^{i}(t, \bar{\kappa}), \\
e_{1}^{i}(t, \bar{\kappa})=\left(e_{1}(t, \bar{\kappa})^{T}, \ldots, e_{i}(t, \bar{\kappa})^{T}, \ldots, e_{p}(t, \bar{\kappa})^{T}\right), \\
e_{2}^{i}(t, \bar{\kappa})=\left(e_{1^{\prime}}(t, \bar{\kappa})^{T}, \ldots, e_{i^{\prime}}(t, \bar{\kappa})^{T}, \ldots, e_{q^{\prime}}(t, \bar{\kappa})^{T}\right) ;
\end{gathered}
$$

under Assumption 6, it holds the following proposition.

Proposition 15. Functions $\delta^{i j}(t, \bar{\kappa}), \delta^{i}(t, \bar{\kappa})$, and $\delta^{j}(t, \bar{\kappa})$ satisfy the following inequality:

$$
\begin{aligned}
E\left[\delta^{i j}\left(t, \bar{\kappa}_{m}\right)\right] \leq & \exp \left[\varepsilon_{\widehat{n}_{\bar{\kappa}_{m}}}\left(\hat{\lambda}_{\bar{\kappa}_{m}}^{i j} T_{\bar{\kappa}_{m}}+\sum_{s_{i}=\bar{\kappa}_{0}}^{\bar{\kappa}_{m-1}} \hat{\lambda}_{s}^{i} T_{s}\right)\right] \\
& \times E\left[\delta^{i}\left(t_{\overline{0}^{i}}, \overline{0}^{i}\right)\right] \\
& +\exp \left[\varepsilon_{\bar{n}_{\bar{\kappa}_{m}}}\left(\hat{\lambda}_{\bar{\kappa}_{m}}^{i j} T_{\bar{\kappa}_{m}}+\sum_{s_{j}=\bar{\kappa}_{0}}^{\bar{\kappa}_{m-1}} \hat{\lambda}_{s}^{j} T_{s}\right)\right] \\
& \times E\left[\delta^{j}\left(t_{\overline{0}^{j}}, \overline{0}^{j}\right)\right]+f\left(t, \bar{\kappa}_{m}\right)+g\left(t, \bar{\kappa}_{m}\right),
\end{aligned}
$$

where $\widehat{\lambda}_{\bar{\kappa}}^{i j}=\max \left\{\alpha(\bar{\kappa})+\lambda_{\bar{\kappa}}^{i j}\right\}, \hat{\lambda}_{\bar{\kappa}}^{i}=\max \left\{\alpha(\bar{\kappa})+\lambda_{\bar{\kappa}}^{i}\right\}, T_{s}=$ $t_{\overline{\mathcal{K}}_{s}}-t_{\overline{\mathcal{K}}_{s-1}}$, and

$$
\begin{aligned}
& g^{i}\left(t, \bar{\kappa}_{m}\right)=\exp \left[\varepsilon_{\bar{n}_{\bar{\kappa}_{m-1}}} \hat{\lambda}_{\bar{\kappa}_{m-1}}^{i}\left(t-t_{\bar{\kappa}_{m-2}}\right)\right] \delta_{2}^{i}\left(t_{\bar{\kappa}_{m-2}}, \bar{\kappa}_{m-2}\right) T_{\bar{\kappa}_{m-2}} \\
& +\exp \left[\varepsilon_{\widehat{\bar{n}}_{\bar{\kappa}_{m-1}}} \hat{\lambda}_{\bar{\kappa}_{m-1}}^{i} T_{\bar{\kappa}_{m-1}}+\varepsilon_{\widehat{n}_{\bar{k}_{m-1}}} \hat{\lambda}_{\bar{\kappa}_{m-2}}^{i} T_{\bar{\kappa}_{m-2}}\right] \\
& \times \delta_{2}^{i}\left(t_{\bar{\kappa}_{m-3}}, \bar{\kappa}_{m-3}\right)+\cdots \\
& +\exp \left[\varepsilon_{\bar{n}_{\bar{k}_{m-1}}} \hat{\lambda}_{\bar{\kappa}_{m-1}}^{i} T_{\bar{\kappa}_{m-1}}+\cdots+\varepsilon_{\bar{n}_{\bar{\kappa}_{1}}} \widehat{\lambda}_{\bar{\kappa}_{1}}^{i} T_{\bar{\kappa}_{1}}\right] \\
& \times \delta_{2}^{i}\left(t_{\bar{\kappa}_{0}}, \bar{\kappa}_{0}\right) \text {, } \\
& g^{j}\left(t, \bar{\kappa}_{m}\right)=\exp \left[\varepsilon_{\bar{\kappa}_{\bar{\kappa}_{m-1}}} \widehat{\lambda}_{\bar{\kappa}_{m-1}}^{j}\left(t-t_{\bar{\kappa}_{m-2}}\right)\right] \delta_{2}^{j}\left(t_{\bar{\kappa}_{m-2}} \bar{\kappa}_{m-2}\right) T_{\bar{\kappa}_{m-2}}
\end{aligned}
$$

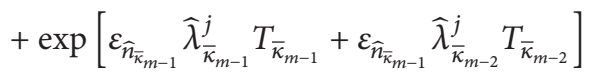

$$
\begin{aligned}
& \times \delta_{2}^{j}\left(t_{\bar{\kappa}_{m-3}}, \bar{\kappa}_{m-3}\right)+\cdots \\
& +\exp \left[\varepsilon_{\widehat{n}_{\bar{\kappa}_{m-1}}} \hat{\lambda}_{\bar{\kappa}_{m-1}}^{j} T_{\bar{\kappa}_{m-1}}+\cdots+\varepsilon_{\widehat{n}_{\bar{k}_{1}^{\prime}}} \widehat{\lambda}_{\bar{\kappa}_{1}^{\prime}}^{j} T_{\bar{\kappa}_{1}^{\prime}}\right] \\
& \times \delta_{2}^{j}\left(t_{\bar{\kappa}_{0^{\prime}}}, \bar{\kappa}_{0^{\prime}}\right) \text {, }
\end{aligned}
$$

where $\bar{\kappa}_{0}$ and $\bar{\kappa}_{0}^{\prime}$ are the initial topologies of $i_{\bar{\kappa}}, j_{\bar{\kappa}}$, respectively. Proof. From criterion (11), it is straightforward that $\left\|\Gamma F^{i}(t)\right\| \leq\left\|X_{\widehat{n}_{\bar{\kappa}}}(t)\right\| ;$ then

$$
\begin{aligned}
\Delta \delta^{i} & =\frac{1}{2} E\left[F^{i}(t)^{T} \Gamma^{T} \Phi_{\bar{\kappa}}^{T} \Phi_{\bar{\kappa}} X_{\widehat{n}_{\bar{\kappa}}}(t)+X_{\widehat{n}_{\bar{\kappa}}}(t)^{T} \Phi_{\bar{\kappa}}^{T} \Phi_{\bar{\kappa}} \Gamma F^{i}(t)\right] \\
& \leq \frac{\alpha(\bar{\kappa})}{2} E\left[X_{\widehat{n}_{\bar{\kappa}}}(t)^{T} \Phi_{\bar{\kappa}}^{T} \Phi_{\bar{\kappa}} X_{\widehat{n}_{\bar{\kappa}}}(t)+X_{\widehat{n}_{\bar{\kappa}}}(t)^{T} \Phi_{\bar{\kappa}}^{T} \Phi_{\bar{\kappa}} X_{\widehat{n}_{\bar{\kappa}}}(t)\right] \\
& \leq \alpha(\bar{\kappa}) E\left[\delta^{i}(t, \bar{\kappa})\right] .
\end{aligned}
$$

Combine (24) with (19); it holds that

$$
\begin{aligned}
E\left[\dot{\delta}^{i j}(t, \bar{\kappa})\right] & \leq \varepsilon_{\widehat{n}_{\bar{\kappa}}}\left(\alpha(\bar{\kappa})+\lambda_{\bar{\kappa}}^{i j}\right) E\left[\delta^{i j}(t, \bar{\kappa})\right] \\
& =\varepsilon_{\bar{n}_{\bar{\kappa}}} \widehat{\lambda}_{\bar{\kappa}}^{i j} E\left[\delta^{i j}(t, \bar{\kappa})\right] ;
\end{aligned}
$$

then $\forall t \in\left[t_{\bar{\kappa}_{m+1}}, t_{\bar{\kappa}_{m}}\right)$,

$$
\begin{aligned}
E\left[\delta^{i j}\left(t, \bar{\kappa}_{m+1}\right)\right] & \leq E\left[\delta^{i j}\left(t_{\bar{\kappa}_{m}}, \bar{\kappa}_{m}\right)\right] \exp \left(\int_{\bar{t}_{\bar{\kappa}_{m}}}^{t} \varepsilon_{\overline{\mathcal{K}}_{m}} \widehat{\lambda}_{\overline{\mathcal{K}}_{m}}^{i j} d s\right), \\
& \leq E\left[\delta^{i j}\left(t_{\overline{\mathcal{K}}_{m}}, \bar{\kappa}_{m}\right)\right] \exp \left[\varepsilon_{\overline{\mathcal{K}}_{m}} \widehat{\lambda}_{\bar{\kappa}_{m}}^{i j}\left(t-t_{\bar{\kappa}_{m}}\right)\right] .
\end{aligned}
$$


Notice that

$$
\begin{aligned}
& E\left[\delta^{i j}\left(t_{\bar{\kappa}_{m}}, \bar{\kappa}_{m}\right)\right] \leq E\left[\delta^{i}\left(t_{\bar{\kappa}_{m}}, \bar{\kappa}_{m}\right)\right]+E\left[\delta^{j}\left(t_{\bar{\kappa}_{m}}, \bar{\kappa}_{m}\right)\right], \\
& E\left[\delta^{i}\left(t_{\bar{\kappa}_{m}}, \bar{\kappa}_{m}\right)\right] \\
& \leq \exp \left[\varepsilon_{\overline{\mathcal{K}}_{m}} \hat{\lambda}_{\overline{\mathcal{K}}_{m}}^{i}\left(t_{\overline{\mathcal{K}}_{m}}-t_{\overline{\mathcal{K}}_{m-1}}\right)\right] \\
& \times E\left[\delta_{i}\left(t_{\bar{\kappa}_{m-1}}, \bar{\kappa}_{m-1}\right)\right] \leq \cdots \\
& \leq \exp \left[\varepsilon_{\bar{\kappa}_{m}} \widehat{\lambda}_{\bar{\kappa}_{m}}^{i}\left(t_{\bar{\kappa}_{m}}-t_{\bar{\kappa}_{m-1}}\right)\right. \\
& +\varepsilon_{\bar{\kappa}_{m-1}} \hat{\lambda}_{\bar{\kappa}_{m-1}}^{i}\left(t_{\bar{\kappa}_{m-1}}-t_{\bar{\kappa}_{m-2}}\right)+\cdots \\
& \left.+\varepsilon_{\bar{\kappa}_{1}} \widehat{\lambda}_{\bar{\kappa}_{1}}^{i}\left(t_{\bar{\kappa}_{1}}-t_{\bar{\kappa}_{0}}\right)\right] E\left[\delta^{i}\left(t_{\bar{\kappa}_{0}}, \bar{\kappa}_{0}\right)\right] \\
& +g^{i}\left(t, \bar{\kappa}_{m}\right), \\
& =\exp \left(\sum_{s=\bar{\kappa}_{0}}^{\bar{\kappa}_{m-1}} \varepsilon_{s} \hat{\lambda}_{s}^{i} T_{s}\right) E\left[\delta^{i}\left(t_{\bar{\kappa}_{0}}, \bar{\kappa}_{0}\right)\right] \\
& +g^{i}\left(t, \bar{\kappa}_{m}\right) \text {, } \\
& E\left[\delta^{j}\left(t_{\bar{\kappa}_{m}}, \bar{\kappa}_{m}\right)\right] \\
& \leq \exp \left[\varepsilon_{\bar{\kappa}_{m}} \hat{\lambda}_{\bar{\kappa}_{m}}^{j}\left(t_{\bar{\kappa}_{m}}-t_{\overline{\mathcal{K}}_{m-1}}\right)\right] \\
& \times E\left[\delta_{j}\left(t_{\bar{\kappa}_{m-1}}, \bar{\kappa}_{m-1}\right)\right] \leq \cdots \\
& \leq \exp \left[\varepsilon_{\bar{\kappa}_{m}} \widehat{\lambda}_{\bar{\kappa}_{m}}^{j}\left(t_{\bar{\kappa}_{m}}-t_{\bar{\kappa}_{m-1}}\right)\right. \\
& +\varepsilon_{\bar{\kappa}_{m-1}} \hat{\lambda}_{\bar{\kappa}_{m-1}}^{j}\left(t_{\bar{\kappa}_{m-1}}-t_{\bar{\kappa}_{m-2}}\right)+\cdots \\
& \left.+\varepsilon_{\bar{\kappa}_{1}} \hat{\lambda}_{\bar{\kappa}_{1}}^{j}\left(t_{\bar{\kappa}_{1}}-t_{\bar{\kappa}_{0}}\right)\right] E\left[\delta^{j}\left(t_{\bar{\kappa}_{0}}, \bar{\kappa}_{0}\right)\right] \\
& +g^{j}\left(t, \bar{\kappa}_{m}\right) \text {, } \\
& =\exp \left(\sum_{s=\bar{\kappa}_{0}}^{\bar{\kappa}_{m}} \hat{\varepsilon}_{s} \hat{\lambda}_{s}^{j} T_{s}\right) E\left[\delta^{j}\left(t_{\bar{\kappa}_{0}}, \bar{\kappa}_{0}\right)\right] \\
& +g^{j}\left(t, \bar{\kappa}_{m}\right) ;
\end{aligned}
$$

from inequalities (26), (27), and (28), it holds the inequality (22). This completes the proof.

Next, the consensus criterion is proposed as follows.

Theorem 16. If subsystem (7) is the GLI-connected and the each attack signal satisfies Assumption 6, then under (4), VSWSN (7) achieves the global average consensus if there is the enough dwell time in the interactional topology $\bar{\kappa}_{m}$, namely,

$$
T_{\bar{\kappa}_{m}}>\max \left\{\frac{1}{\hat{\lambda}_{\bar{\kappa}_{m}}^{i j}}\left(-\sum_{s=\bar{\kappa}_{0}}^{\bar{\kappa}_{m-1}} \hat{\lambda}_{s}^{i} T_{s}\right), \frac{1}{\hat{\lambda}_{\bar{\kappa}_{m}}^{i j}}\left(-\sum_{s=\bar{\kappa}_{0^{\prime}}}^{\bar{\kappa}_{m-1}} \hat{\lambda}_{s}^{j} T_{s}\right)\right\},
$$

and $\varepsilon_{\widehat{n}_{\bar{\kappa}}}$ satisfies (16).

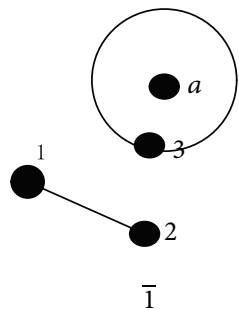

(a)

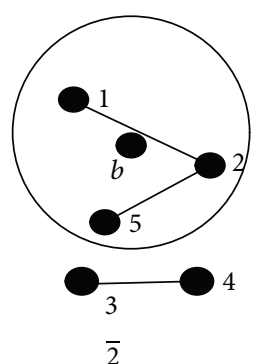

(b)

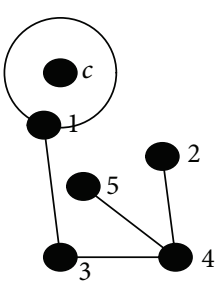

$\overline{3}$

(c)
FIGURE 1: Two node attached components of nodes 1 and 3 in VSWSN.

Proof. For inequality (29), it follows that $\widehat{\lambda}_{\bar{\kappa}_{m}}^{i j}<0, \widehat{\lambda}_{\bar{\kappa}_{m}}^{i} \leq 0$, based on Proposition 15, and if each attack signal satisfies Assumption 6, then

$$
\lim _{T_{\bar{\kappa}_{m}} \rightarrow \infty} E\left[\delta^{i j}(t, \bar{\kappa})\right]=0 ;
$$

this completes the proof.

Remark 17. In $E\left[\delta^{i j}\left(t, \bar{\kappa}_{m+1}\right)\right] \leq E\left[\delta^{i j}\left(t_{\bar{\kappa}_{m}}, \bar{\kappa}_{m}\right)\right] \exp \left[\varepsilon_{\bar{\kappa}_{m}} \hat{\lambda}_{\bar{\kappa}}^{i j}(t-\right.$ $\left.t_{\bar{\kappa}_{m}}\right)$ ], even though $\widehat{\lambda}_{\bar{\kappa}}^{i j}<0$, it cannot ensure that the system achieves the average consensus; see simulation results of Example 2. In addition, if adaptive parameter (16) is utilized, whatever $E\left[\delta^{i j}\left(t_{\bar{\kappa}_{m}}, \bar{\kappa}_{m}\right)\right]$ tends to infinite, the system achieves the consensus, and this result is different from the literature [7].

Remark 18. Theorem 16 shows that under the tolerable jamming attacks, if VSWSN is the GLI-connected and the value of $\varepsilon_{\widehat{n}_{\overrightarrow{\mathrm{V}}}}$ is chosen appropriately, then VSWSN achieves the consensus. The numerical example of the following section shows the reliability.

\section{Numerical Example}

Example 1. Suppose that VSWSN (7) is composed of the following two node attached components:

$$
\begin{aligned}
& C_{\bar{\kappa}}^{1}=\left(V^{1}(\kappa), E^{1}(\kappa), A^{1}(\kappa)\right), \\
& C_{\bar{\kappa}}^{3}=\left(V^{3}(\kappa), E^{3}(\kappa), A^{3}(\kappa)\right),
\end{aligned}
$$

where $T_{0}(t)=\{\overline{1}, \overline{2}, \overline{3}\}$, the figure of $T_{0}(t)$ is shown in Figure 1 , $a, b, c$ refer to the attack signals under the different topologies, and the circles refer to the sensible regions. For convenience, the topology indexes of nodes are dropped in the following. Suppose that

$$
\begin{gathered}
V^{1}(\overline{1})=\{1,2\}, \quad V^{3}(\overline{2})=\{1,2,5\}, \\
V^{1}(\overline{3})=\{1,2,3,4,5\}, \\
V^{3}(\overline{1})=\{3\}, \quad V^{3}(\overline{2})=\{3,4\}, \\
V^{3}(\overline{3})=\{1,2,3,4,5\} .
\end{gathered}
$$



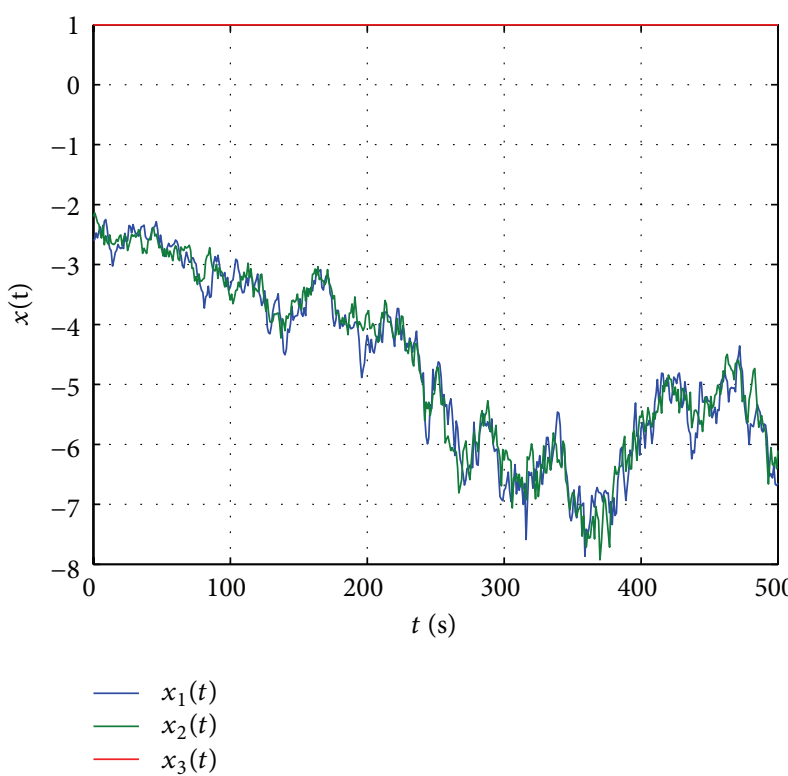

(a) The dynamic states of all sensors

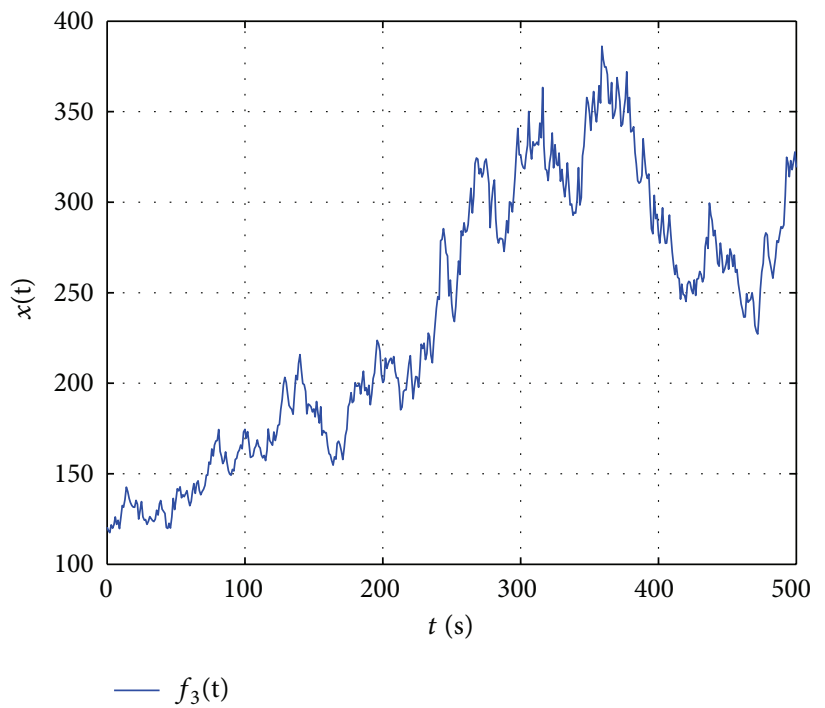

(c) State of the attack signal, where $f_{3}(t)=f_{a}(t)$
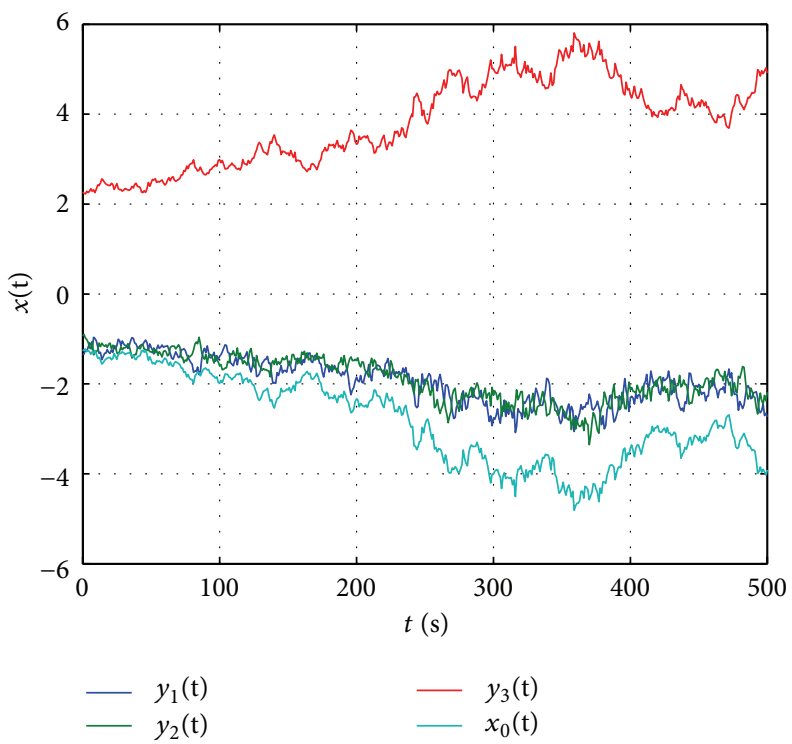

(b) The errors among the average values and states of all nodes, where $e^{i}(t)=y_{i}(t), i=1,2,3$

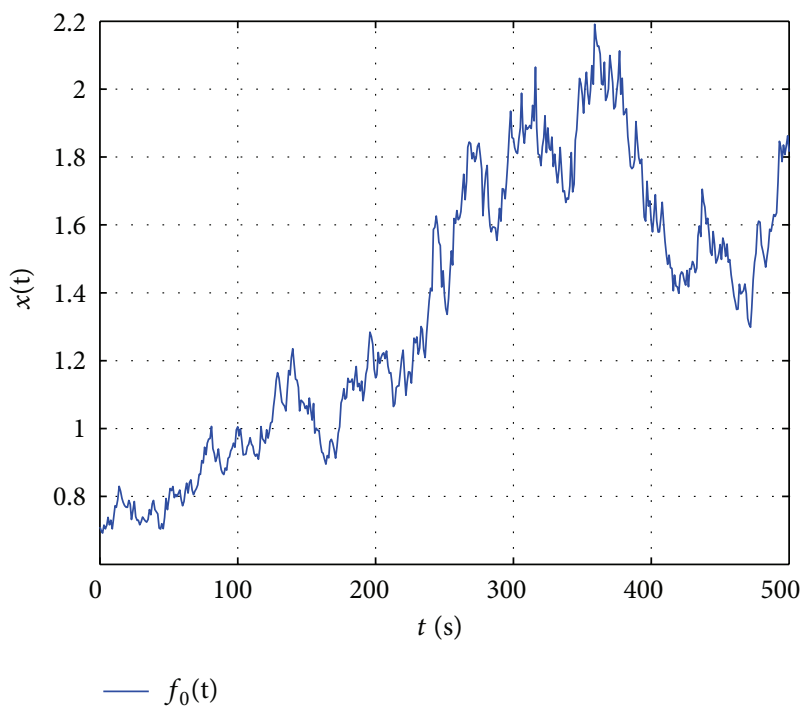

(d) The update law of $\varepsilon_{\bar{\kappa}_{1}}$, where $f_{0}(t)=\varepsilon_{\bar{\kappa}_{1}}$

Figure 2: The dynamic states of $X_{k}, Y_{k}$, jamming attack signal and the update law of $\varepsilon_{\bar{\kappa}_{1}}$ in topology $\overline{1}$.

The related matrices are listed as follows:

$$
\begin{gathered}
L^{1}(\overline{1})=\left[\begin{array}{cc}
1 & -1 \\
-1 & 1
\end{array}\right], \\
L^{1}(\overline{2})=\left[\begin{array}{ccc}
1 & -1 & 0 \\
-1 & 2 & -1 \\
0 & -1 & 1
\end{array}\right], \\
L^{1}(\overline{3})=\left[\begin{array}{ccccc}
1 & -1 & 0 & 0 & 0 \\
-1 & 2 & 0 & 0 & -1 \\
0 & 0 & 1 & -1 & 0 \\
0 & 0 & -1 & 1 & 0 \\
0 & -1 & 0 & 0 & 1
\end{array}\right],
\end{gathered}
$$

$$
\begin{gathered}
L^{3}(\overline{2})=\left[\begin{array}{cc}
1 & -1 \\
-1 & 1
\end{array}\right], \\
L^{3}(\overline{3})=L^{1}(\overline{3}) .
\end{gathered}
$$

Under protocol (4), the dynamic state of the node attached component is given by (5); the related parameters are listed below.

In topology $\overline{1}$, the second largest eigenvalue of $-L^{1}(\overline{1})$ and $-L^{3}(\overline{1})$ is -2 ; the jamming attack signal is $f_{a}(t)=2\left\|x^{1}(t, \overline{1})\right\|$, $\alpha(\overline{1})=1$, the measured signals are $f_{13}(t)=f_{23}(t)=f_{a}(t)$, and Assumption 6 cannot be satisfied; node 3 falls in sleeping 

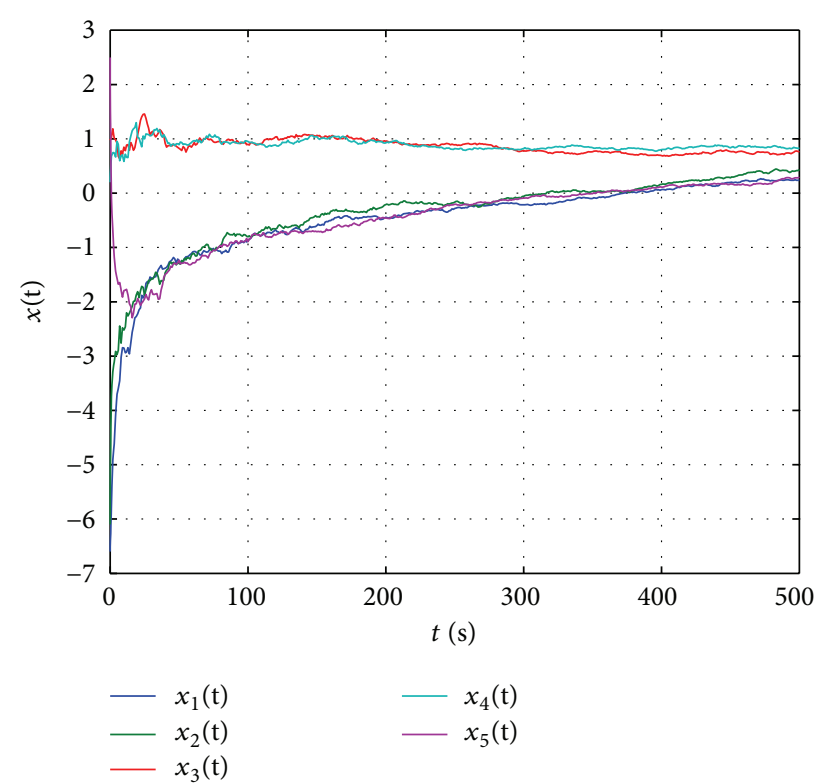

(a) The dynamic states of all sensors

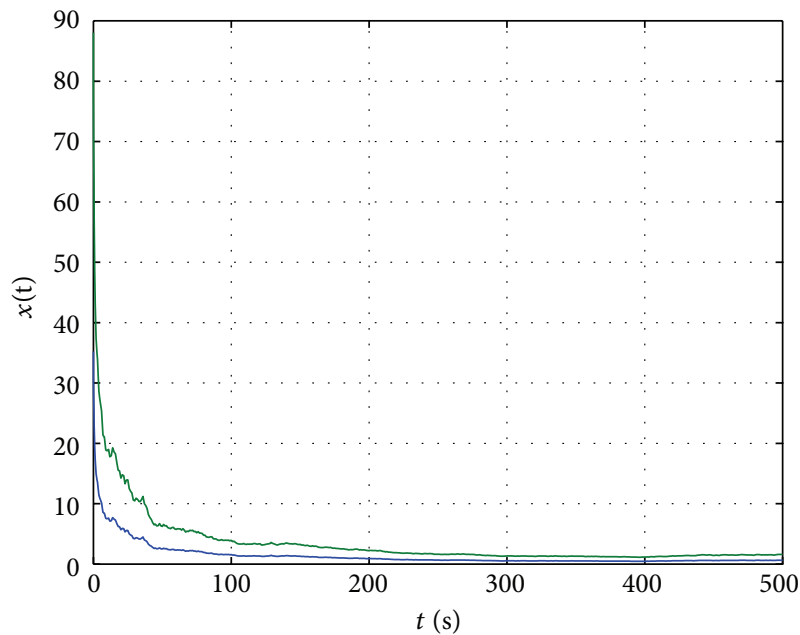

$\begin{aligned}- & s_{1}(\mathrm{t}) \\ - & s_{2}(\mathrm{t})\end{aligned}$

(c) $s_{1}(t)$ is the attack signal, $s_{2}(t)=\varepsilon_{\bar{\kappa}_{2}}$

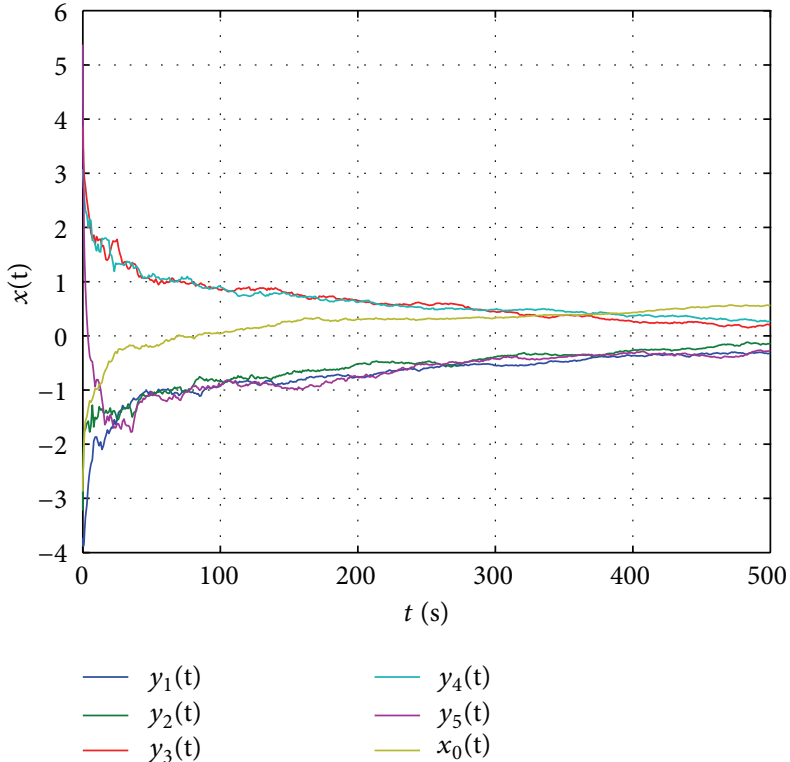

(b) The errors among the average values and states of all nodes, where $e^{i}(t)=y_{i}(t), i=1,2,3,4,5$

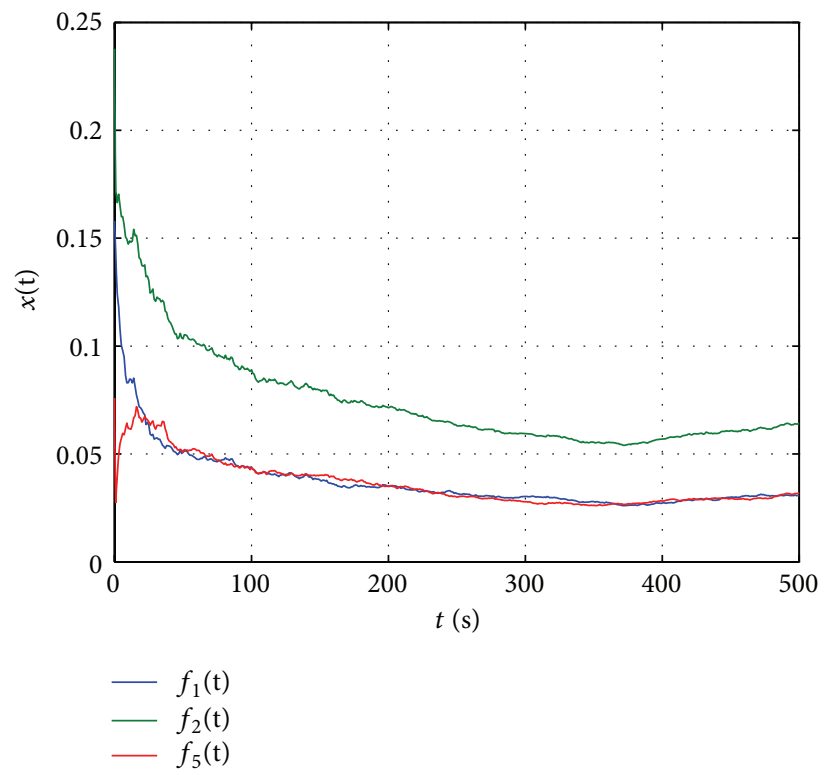

(d) $f_{1}(t), f_{2}(t), f_{5}(t)$ stand the measured attack signals by nodes 1,2 , and 5 , respectively

FIgURE 3: The dynamic states of $X_{k}, Y_{k}$, attack signals, and the update law of $\varepsilon_{\bar{\kappa}_{2}}$ in topology $\overline{2}$.

(see topology $\overline{1}$ of Figure 1). Taking $c=0.1$, suppose that $\varepsilon_{\bar{\kappa}}$ satisfies (16); the simulation results are shown in Figure 2.

In topology $\overline{2}$, according to carrier sense, node 3 is awakened, node 5 joined the node attached component of 1 , and node 4 joined the node attached component of 3 (see topology $\overline{2}$ of Figure 1). For this topology, the second largest eigenvalue of $-L^{1}(\overline{2})$ and $-L^{3}(\overline{2})$ is -1 . Suppose that the jamming attack signal is $f_{b}(t)=\left\|x^{2}(t, \overline{2})\right\|, \alpha(\overline{1})=0.2$, the measured signals are $f_{13}(t)=f_{23}(t)=0.01 f_{b}(t)$, and
Assumption 6 is satisfied; taking $c=0.1$, suppose that $\varepsilon_{\bar{\kappa}}$ satisfies (16); the simulation results are shown in Figure 3.

In topology $\overline{3}$, two node attached components are merged (see topology $\overline{3}$ of Figure 1). For this topology, the second largest eigenvalue of $-L^{1}(\overline{3})$ is -1 . Suppose that the jamming attack signal is $f_{c}(t)=\left\|x^{3}(t, \overline{3})\right\|, \alpha(\overline{1})=0.3$, the measured signals are $f_{13}(t)=f_{31}(t)=0.01 \times\left[(4 / 25) f_{c}(t)\right]$, and Assumption 6 is satisfied; taking $c=0.1$, suppose that $\varepsilon_{\bar{\kappa}}$ satisfies (16); the simulation results are shown in Figure 4. 


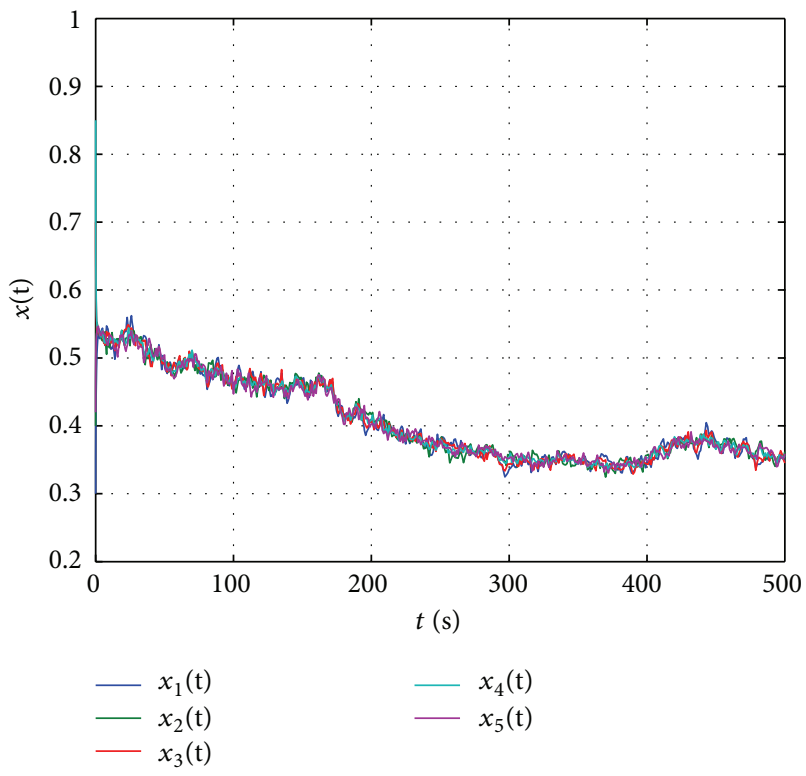

(a) The dynamic states of all sensors

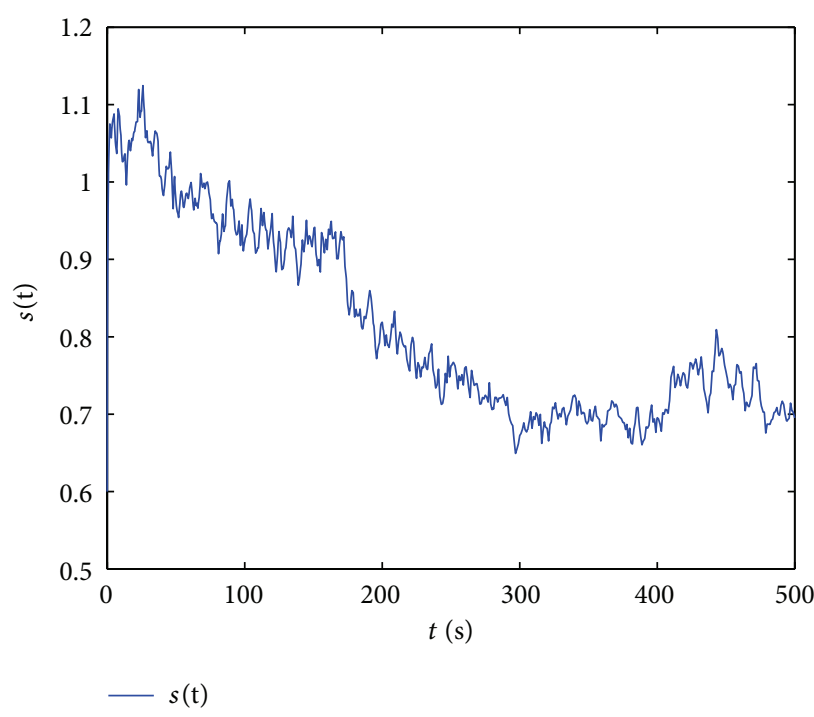

(c) The state of the jamming attack signal, where $s(t)=f_{c}(t)$

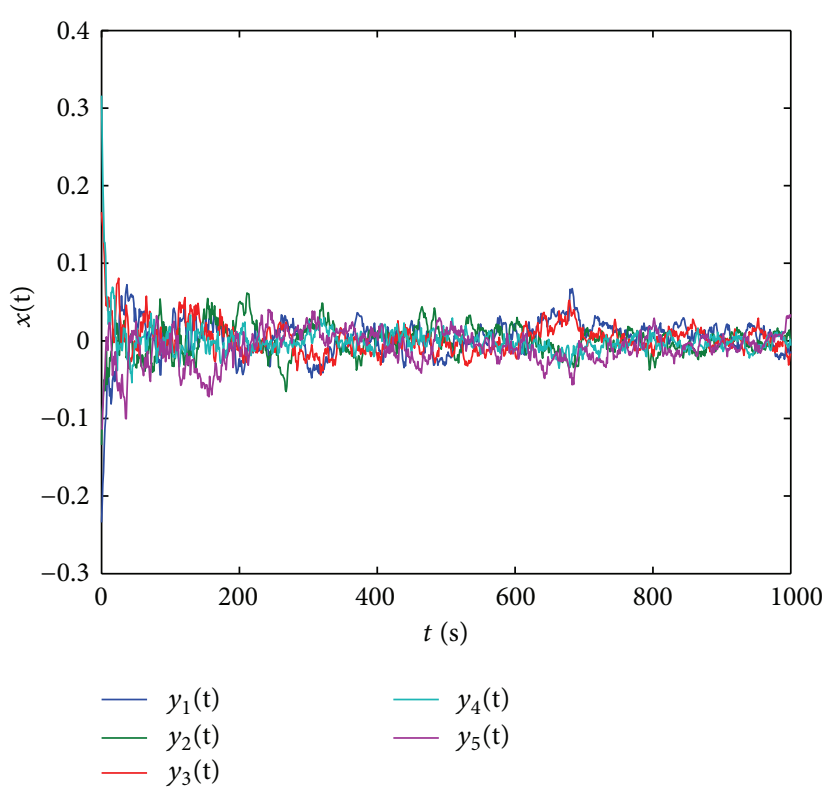

(b) The errors among the average values and states of all nodes, where $e^{i}(t)=y_{i}(t), \quad i=1,2,3,4,5$

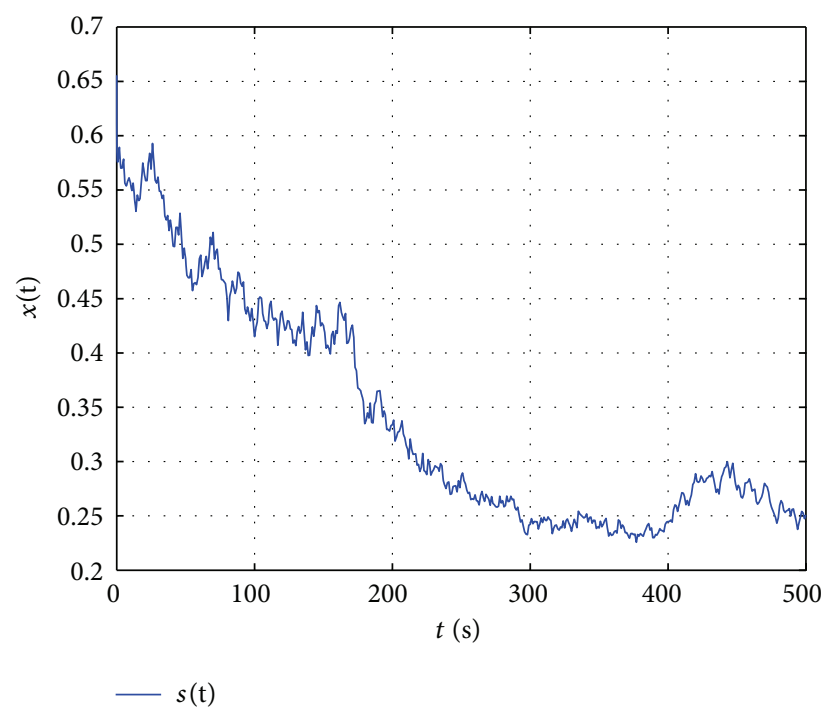

(d) The update law of $\varepsilon_{\bar{\kappa}_{3}}$, where $s(t)=\varepsilon_{\bar{\kappa}_{3}}$

FIgURE 4: The dynamic states of $X_{k}, Y_{k}$, jamming attack signal, and the update law of $\varepsilon_{\bar{\kappa}_{3}}$ in topology $\overline{3}$.

Example 2. Following Example 1, if $\varepsilon_{\bar{\kappa}}$ is replaced by the gain function $a(t)=\log (t+2) /(t+2)$ studied in [7], the system with topology $\overline{3}$ cannot achieve the consensus; the simulation results are shown in Figure 5.

\section{Conclusion}

This paper has investigated the consensus problem of VSWSN under the tolerable jamming attacks. It has disclosed the relations among the attack power, initial values of the newly joined nodes, dwell time, and GLI-connected topology. According to the errors of the node attached components, the adaptive parameters were provided; then the adaptive consensus protocol was obtained, and the designed protocol ensures that the system achieves the consensus whatever the values of the newly joined nodes. The obtained results in this paper have extended some existing results which are associated with the fixed node set system. In fact, according to the attack power, this paper has provided a sleep method of VSWSN when the system encounters with the jamming attacks. Finally, simulation results have shown the effectiveness of the obtained results.

For the future research, relations among the time delays of multiple hop-relays, accumulated errors, and the consensus will be considered. 


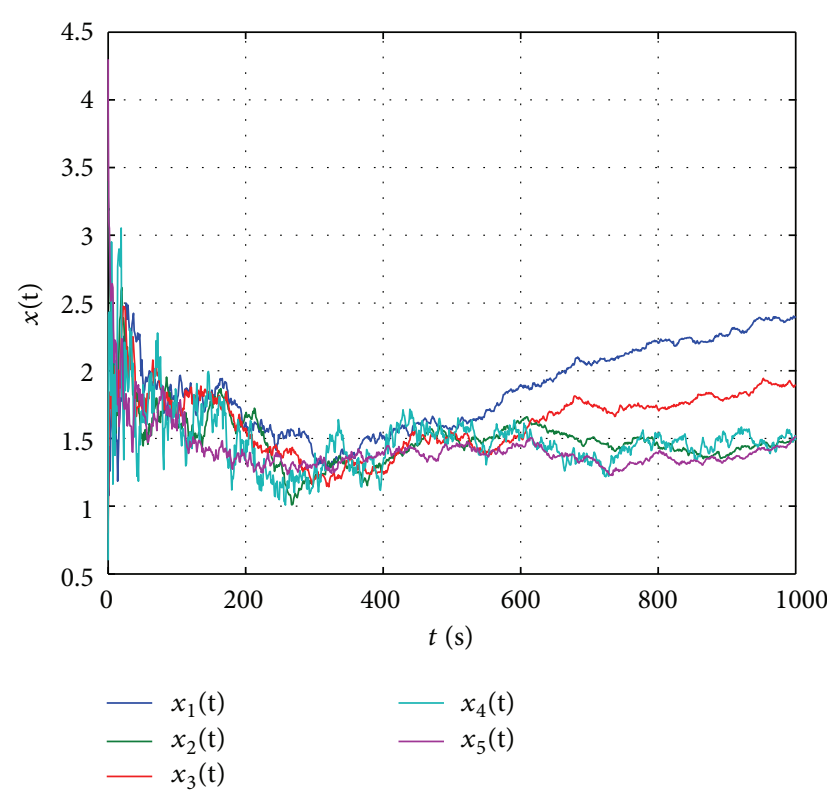

(a) The dynamic states of all sensors

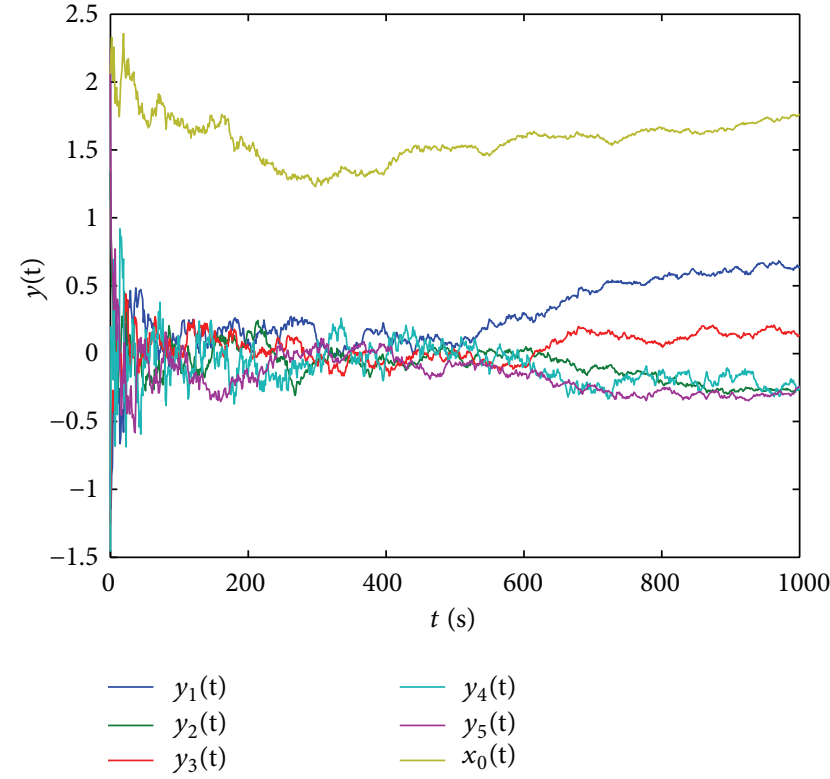

(b) The errors among the average values and states of all nodes, where $e^{i}(t)=y_{i}(t), i=1,2,3,4,5$

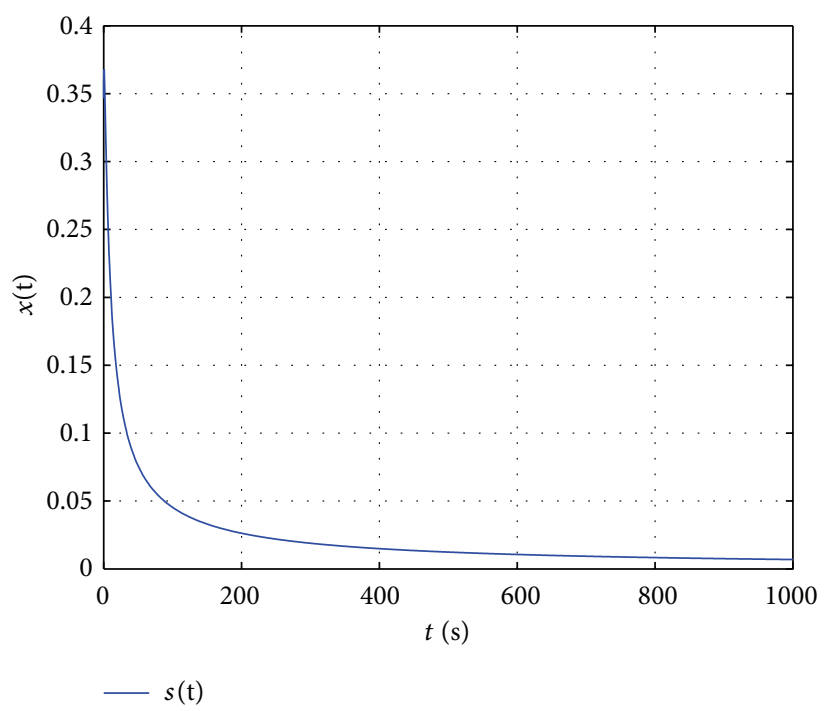

(c) Curves of the gain function $s(t)=\log (t+2) /(t+2)$

Figure 5: The dynamic states of $X_{k}, Y_{k}$, and gain function $s(t)$ of Example 2.

\section{Acknowledgment}

This work is supported by Cultivation Fund of Taizhou University (2013PY09).

\section{References}

[1] J. Yick, B. Mukherjee, and D. Ghosal, "Wireless sensor network survey," Computer Networks, vol. 52, no. 12, pp. 2292-2330, 2008.

[2] I. F. Akyildiz, W. Su, Y. Sankarasubramaniam, and E. Cayirci, "Wireless sensor networks: a survey," Computer Networks, vol. 38, no. 4, pp. 393-422, 2002.
[3] D. Zhao, Z. An, and Y. Xu, "Time synchronization in wireless sensor networks using max and average consensus protocol," International Journal of Distributed Sensor Networks, vol. 2013, Article ID 192128, 10 pages, 2013.

[4] Z.-G. Wu, J. H. Park, H. Su, B. Song, and J. Chu, "Exponential synchronization for complex dynamical networks with sampled-data," Engineering and Applied Mathematics, vol. 349, no. 9, pp. 2735-2749, 2012.

[5] Z.-G. Wu, J. H. Park, H. Su, and J. Chu, "Discontinuous Lyapunov functional approach to synchronization of time-delay neural networks using sampled-data," Nonlinear Dynamics, vol. 69, no. 4, pp. 2021-2030, 2012.

[6] R. Olfati-Saber and R. M. Murray, "Consensus problems in networks of agents with switching topology and time-delays," 
Transactions on Automatic Control, vol. 49, no. 9, pp. 1520-1533, 2004.

[7] T. Li and J.-F. Zhang, "Mean square average-consensus under measurement noises and fixed topologies: necessary and sufficient conditions," Automatica, vol. 45, no. 8, pp. 1929-1936, 2009.

[8] W. Zhou, J. Mou, T. Wang, C. Ji, and J. Fang, "Quasi-average mean square consensus for wireless sensor networks under three topologies with respect to sleeping-awaking method," Optimal Control Applications and Methods, vol. 34, no. 4, pp. 379-395, 2013.

[9] N. Abaid and M. Porfiri, "Leader-follower consensus over numerosity-constrained random networks," Automatica, vol. 48, no. 8, pp. 1845-1851, 2012.

[10] L. Ding, Q.-L. Han, and G. Guo, "Network-based leaderfollowing consensus for distributed multi-agent systems," Automatica, vol. 49, no. 7, pp. 2281-2286, 2013.

[11] Y. Hu, H. Su, and J. Lam, "Adaptive consensus with a virtual leader of multiple agents governed by locally Lipschitz nonlinearity," International Journal of Robust and Nonlinear Control, vol. 23, no. 9, pp. 978-990, 2013.

[12] Z. Ji, H. Lin, and H. Yu, "Leaders in multi-agent controllability under consensus algorithm and tree topology," Systems \& Control Letters, vol. 61, no. 9, pp. 918-925, 2012.

[13] W. Zhou, J. Mou, T. Wang, C. Ji, and J. Fang, "Targetsynchronization of the distributed wireless sensor networks under the same sleeping-awaking method," Journal of the Franklin Institute, vol. 349, no. 6, pp. 2004-2018, 2012.

[14] F. Fagnani and S. Zampieri, "Randomized consensus algorithms over large scale networks," IEEE Journal on Selected Areas in Communications, vol. 26, no. 4, pp. 634-649, 2008.

[15] M. Challenger, E. Haytaoglu, G. Tokatli, O. Dagdeviren, and K. Erciyes, "A hybrid distributed mutual exclusion algorithm for cluster-based systems," Mathematical Problems in Engineering, vol. 2013, Article ID 703414, 15 pages, 2013.

[16] T. Botmart and P. Niamsup, "Delay-dependent synchronization for complex dynamical networks with interval time-varying and switched coupling delays," Journal of Applied Mathematics, vol. 2013, Article ID 367457, 16 pages, 2013.

[17] Z. Li, W. Ren, X. Liu, and L. Xie, "Distributed consensus of linear multi-agent systems with adaptive dynamic protocols," Automatica, vol. 49, no. 7, pp. 1986-1995, 2013.

[18] A. Chapman and M. Mesbahi, "Semi-autonomous consensus: network measures and adaptive trees," IEEE Transactions on Automatic Control, vol. 58, no. 1, pp. 19-31, 2013.

[19] Z. Li, W. Ren, X. Liu, and M. Fu, "Consensus of multi-agent systems with general linear and Lipschitz nonlinear dynamics using distributed adaptive protocols," Institute of Electrical and Electronics Engineers, vol. 58, no. 7, pp. 1786-1791, 2013.

[20] H. Wang and Y. Guo, "Consensus on scale-free network," in Proceedings of the Amercia Control Conference (ACC '08), pp. 748-752, Seattle, Wash, USA, June 2008.

[21] H. Yang, L. Lan, and S. Zhang, "Consensus of synchronizationpreferential scale free networks," Physics Procedia, vol. 3, no. 5, pp. 1913-1920, 2010.

[22] J. Mou, W. Zhou, T. Wang, C. Ji, and D. Tong, "Consensus of the distributed varying scale wireless sensor networks," Mathematical Problems in Engineering, vol. 2013, Article ID 862518, 9 pages, 2013.

[23] X. F. Wang and G. Chen, "Synchronization in scale-free dynamical networks: robustness and fragility," IEEE Transactions on Circuits and Systems I, vol. 49, no. 1, pp. 54-62, 2002.
[24] Y.-W. Wang, H. O. Wang, J.-W. Xiao, and Z.-H. Guan, "Synchronization of complex dynamical networks under recoverable attacks," Automatica, vol. 46, no. 1, pp. 197-203, 2010.

[25] A. Cerpa and D. Estrin, "ASCENT: adaptive self-configuring sensor networks topologies," IEEE Transactions on Mobile Computing, vol. 3, no. 3, pp. 272-285, 2004.

[26] R. Horn and C. Johnson, Matrix Analysis, Cambridge University, New York, NY, USA, 1995. 


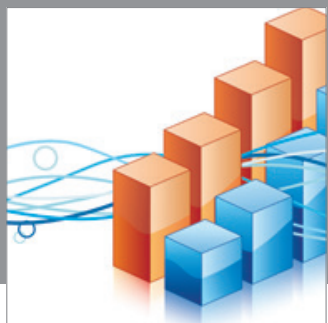

Advances in

Operations Research

mansans

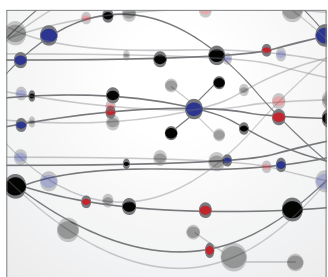

The Scientific World Journal
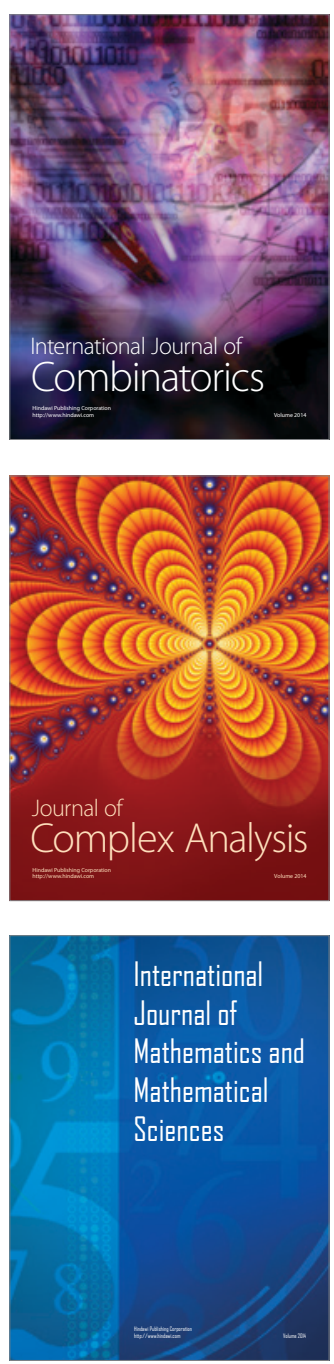
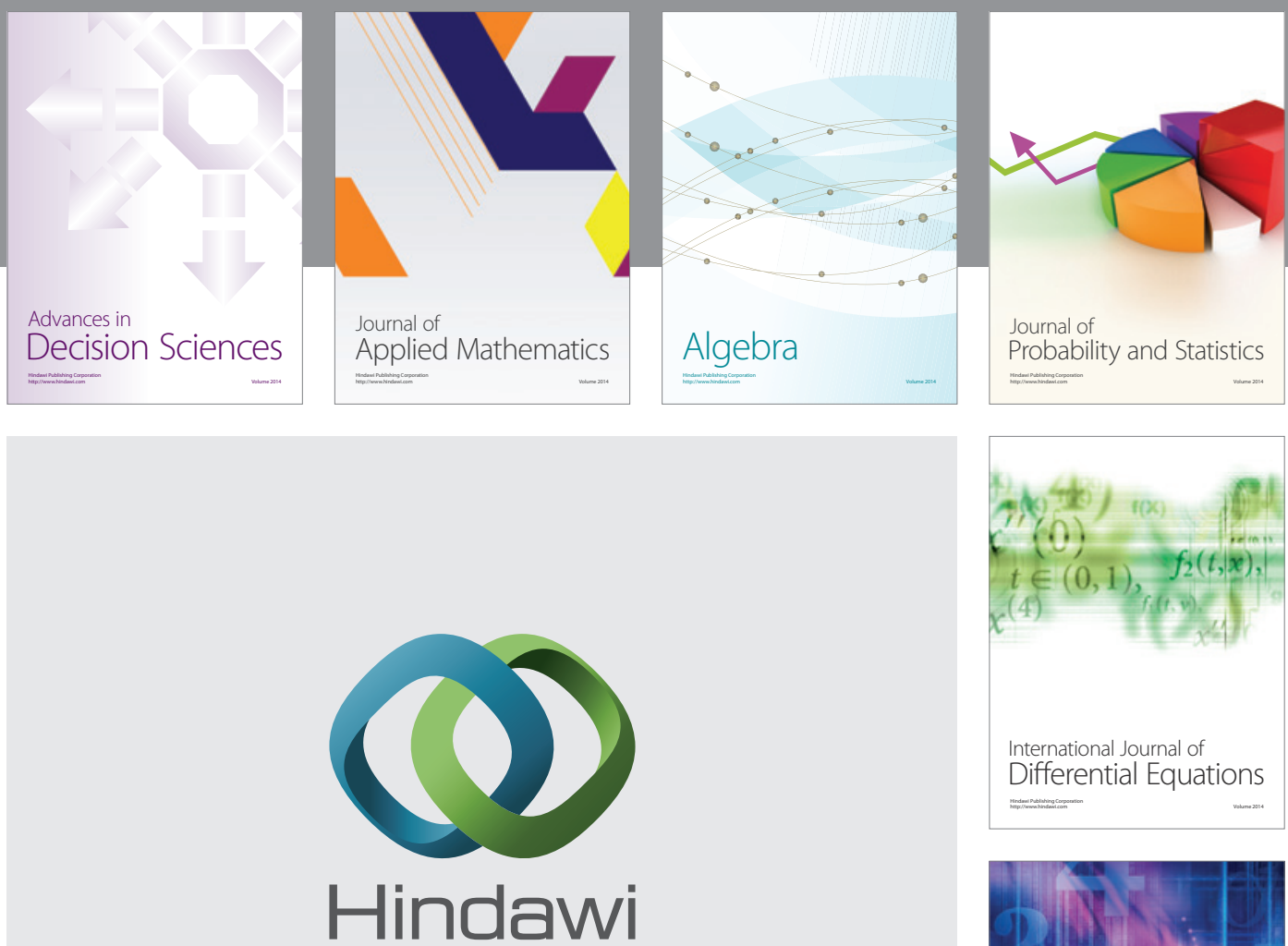

Submit your manuscripts at http://www.hindawi.com
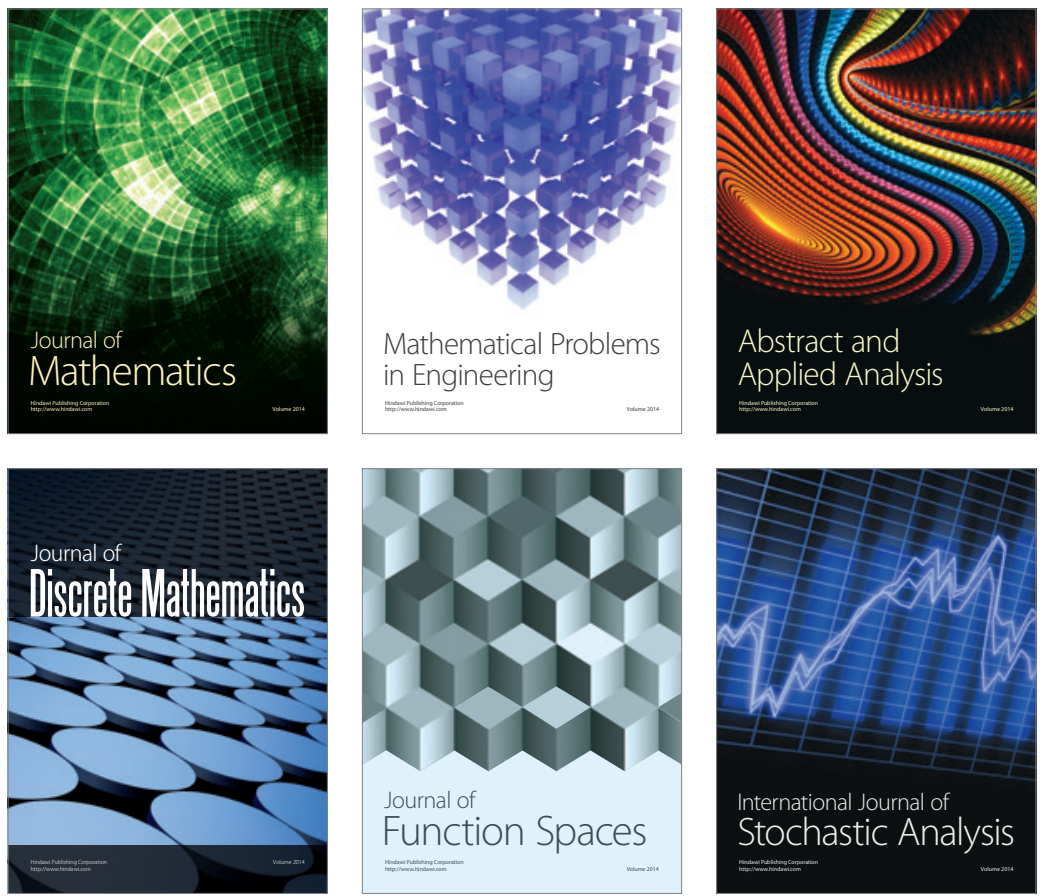

Journal of

Function Spaces

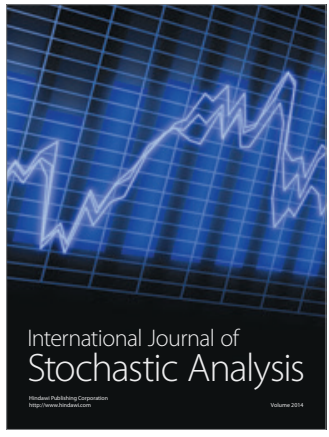

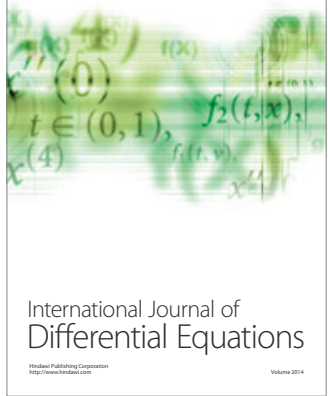
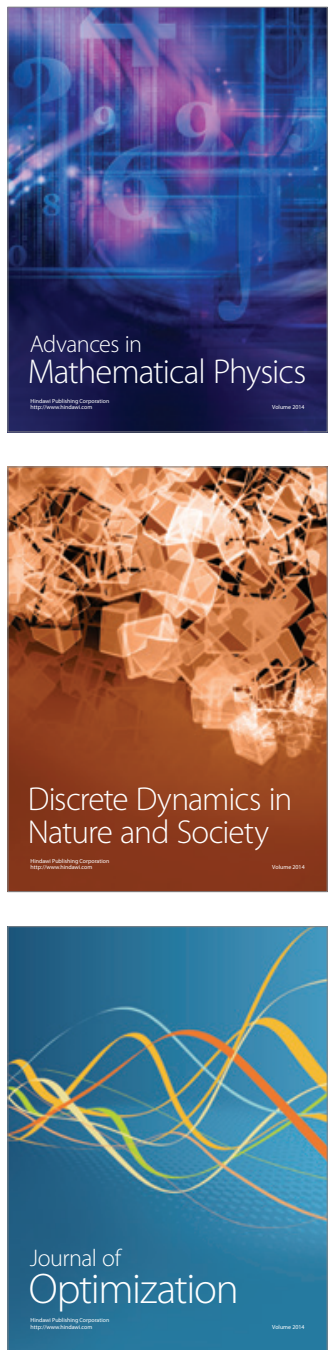